\title{
Análisis 3D de la deformación y cinemática de la Fosa Mesoamericana en la confluencia entre las placas de Rivera y Cocos con las placas Caribe y Norteamericana
}

\author{
Strain 3D analysis and kinematics of the Middle American Trench in the junction of the Rivera and \\ Cocos plates with the North-American and Caribbean plates
}

Jorge Luis Giner-Robles ${ }^{\text {** }}$, Raúl Pérez-López ${ }^{2}$, Javier Elez ${ }^{3}$, Pablo Silva ${ }^{3}$, Elvira Roquero ${ }^{4}$, Adrià Ramos ${ }^{2}$, Garolina Canora ${ }^{1}$, Emilio Rodríguez-Escudero ${ }^{1}$, Miguel Ángel Rodríguez-Pascua ${ }^{2}$

\begin{abstract}
Departamento de Geología y Geoquímica, Facultad de Ciencias, Universidad Autónoma de Madrid, Ciudad Universitaria de Cantoblanco s/n, 28049 Madrid, España.
\end{abstract}

${ }^{2}$ Instituto Geológico y Minero de España, Ríos Rosas, 23, 28003 Madrid, España.

${ }^{3}$ Departamento de Geología, Universidad de Salamanca, Escuela Politécnica Superior de Ávila, Facultad de Ciencias, Hornos Caleros, 50, 05003 Ávila, España.

${ }^{4}$ Departamento de Edafología. Escuela Técnica Superior de Ingenieros Agrónomos, Universidad Politécnica de Madrid, Ciudad Universitaria s/n, 28040 Madrid, España.

* Autor para correspondencia: (J. L. GinerRobles)jorge.giner@uam.es

\begin{abstract}
Cómo citar este artículo: Rodríguez-Escudero, E., Rodríguez-Pascua, M.A., 2021, Análisis 3D de la deformación y con las placas Caribe y Norteamericana: 73 (2), A150121. http://dx.doi.org/10.18268/ BSGM2021v73n2a150121

Manuscrito recibido: 17 de Septiembre de 2020 Manuscrito corregido: 30 de Diciembre de 2020 Manuscrito aceptado: 7 de Enero de 2021
\end{abstract}

Giner-Robles, J.L., Pérez-López, R., Elez, J., Silva, P., Roquero, E., Ramos, A., Canora, C., cinemática de la Fosa Mesoamericana en la confluencia entre las placas de Rivera y Cocos Boletín de la Sociedad Geológica Mexicana,

La revisión por pares es responsabilidad de la Universidad Nacional Autónoma de México.

\section{RESUMEN}

Este trabajo propone un modelo cinemático 3D de la confluencia de las placas tectónicas Norteamericana y Pacífica con las placas de Rivera y Cocos, centrado en la zona de convergencia de la Fosa Mesoamericana en el área de México. A partir del análisis de más de 1300 mecanismos focales de terremotos $(M \geq 5.5)$ obtenidos de la base de datos en abierto del programa Global Centroid-Moment-Tensor (CMT), se han aplicado diversas técnicas de geología estructural para obtener la distribución espacial de los tensores de deformación $\left(\mathrm{e}_{\mathrm{v}}, \mathrm{e}_{\mathrm{x}}, \mathrm{e}_{\mathrm{z}}\right)$ y del factor de forma $\left(\mathrm{k}^{\prime}\right)$ de dichos tensores. Estas técnicas consisten en el estudio de los Diedros Rectos de los mecanismos focales y de la aplicación del Modelo de Deslizamiento, basados en la cinemática del deslizamiento sobre el plano de falla teórico asociado a cada sismo. Este análisis permite realizar un estudio tridimensional de la deformación mediante el agrupamiento espacial 3D de los diferentes tensores (análisis cluster), agrupando zonas homogéneas en estilos de deformación, junto con los diagramas triangulares de clasificación de mecanismos focales (Kaverina) en relación con la geometría de la subducción y de la presencia de flujos mantélicos. También se han calculado perfiles profundos de la deformación y del factor de forma ( $\left.k^{\prime}\right)$ en relación a la geometría de la subducción y las principales estructuras tectónicas. Los resultados muestran una zonación 3D influenciada por el ángulo de subducción y el aumento de la velocidad de convergencia hacia el SE, en relación al acoplamiento mecánico de la subducción, apareciendo menos terremotos inversos en la convergencia de la Placa de Rivera con la Placa Norteamericana, frente a la Placa de Cocos con la Placa Norteamericana. La geometría 3D propuesta es congruente con la distribución espacial de grandes terremotos, el ángulo de subducción, la presencia de corrientes mantélicas a $100 \mathrm{~km}$ de profundidad y la presencia de terremotos en fallamiento normal. Este modelo también podría explicar la presencia de terremotos anómalos como terremotos lentos y silenciosos en el Gap de Guerrero.

Palabras clave: mecanismo focal, Inversión, tensor deformación, k', México.

\section{ABSTRACT}

In this work, we propose a kinematic model based on the strain 3D spatial distribution from focal mechanism solutions of instrumental earthquakes located along the Middle American Trench, in the convergence zone of Rivera and Cocos plates with North-American and Caribbean tectonic plates. We have used more than 1300 focal mechanisms of earthquakes $(M \geq 5.5)$, from the Global Centroid-Moment-Tensor (CMT) online global program to perform a cluster analysis of the spatial distribution of the tectonic strain tensor $\left(e, e_{2}, e_{2}\right)$ and its shape ( $\left.k^{\prime}\right)$. Accordingly, we have applied the Right Dihedral Method to a population of geographically clustered focal mechanisms, as well as the Slip Method, based on the slip data on the theoretical fault plane, for determining the extensional, compressive, and strike-slip zones, in correspondence with the principal tectonic features. Furthermore, we have realized 14 depth profiles across the Middle American Trench (MAT) of the shape of the strain tensor and ternary Kaverina diagrams for constraining the tectonic deformation and developing a 3D model of the kinematics in the region. This analysis depicts a zonation in relation with the changing subduction angle of the slab and an increase of the convergence rate towards the SE, resulting from the mechanical coupling of the subduction, and showing less thrust earthquakes at the Rivera-North American convergence zone than at the Cocos-North American one. The proposed 3D geometry is in good agreement with the spatial distribution of large earthquakes, the slab dip angle, the presence of mantle currents at a $100 \mathrm{~km}$ depth and the occurrence of normal-type earthquakes. This model could also account for the presence of anomalous earthquakes such as slow and silent earthquakes in the Guerrero Gap.

Keywords: focal mechanism, Inversion, strain tensor, $k^{\prime}$, Mexico. 


\section{Introducción}

El crecimiento continuo en el tiempo de las bases de datos de grandes terremotos (magnitud superior a M 6), aumenta el conocimiento sobre su ciclo sísmico y la ocurrencia de grandes terremotos con capacidad de liberar energía por encima de magnitud M 8.5, e incluso de mega-terremotos de magnitud superior a M 9 (i.e. Ekström et al., 2012). En aquellos límites de placa con elevado potencial sísmico destructivo, tanto en las zonas con elevada velocidad de convergencia (c.a. decenas de centímetros año), como en zonas intraplaca con grandes fallas superficiales con capacidad de disparar terremotos de magnitud M 7, el análisis mediante técnicas estadísticas de campos de esfuerzo/deformación (Marret y Peacock, 1999) permite mejorar los estudios de peligrosidad sísmica al disminuir la incertidumbre sobre las fuentes sismogéneticas y sus terremotos máximos. El estudio de la deformación tectónica a lo largo de las grandes estructuras como límites de placas de subducción, grandes zonas en extensión y límites en desgarre, permite llevar a cabo el estudio de la cinemática de dicha deformación, lo que a su vez nos lleva al estudio de la geometría de dichas estructuras (Marret y Peacock, 1999). Además, dicho estudio parte de los datos observados, lo que confiere bastante realismo a los datos obtenidos, describiendo una geometría de la deformación más exacta y que por lo tanto, disminuye la incertidumbre a la hora de hacer modelos que expliquen la presencia de flujos mantélicos o cambios en la geometría de subducción (Giner-Robles et al., 2008, 2009), así como el estudio de propiedades complejas y geometrías fractales que puedan entrar en relación con el vulcanismo reciente de islas oceánicas en zonas tectónicamente activas (Pérez-López et al., 2007).

Dentro de los análisis estadísticos del campo de esfuerzo/deformación a partir del estudio del vector deslizamiento sobre el plano de falla (e.g. $\mathrm{Xu}, 2004)$, en este trabajo se ha llevado a cabo el estudio de la distribución 3D de la deformación a partir de la distribución espacial del factor de forma del tensor de deformación $\left(\mathrm{k}^{\prime}=\mathrm{e}_{\mathrm{y}} / \mathrm{e}_{\mathrm{z}}\right.$, donde $\mathrm{e}_{\mathrm{y}}$ es la dirección de máximo acortamiento horizontal y e es el acortamiento vertical), obtenido a partir de los mecanismos focales de terremotos (e.g. De Vicente, 1987; Herraiz et al., 2000; Giner Robles et al., 2003a, 2003b, 2008; De Vicente et al., 2008; Olaiz et al., 2009), junto con la definición de la tipología del terremoto y la geometría del movimiento de la falla asociada, obtenida a partir del diagrama ternario de Kaverina (e.g. Álvarez-Gómez et al., 2019). El diagrama ternario de Kaverina es una proyección equiareal de los ejes $\mathrm{P}$, $\mathrm{T}$ y $\mathrm{N}$ de los mecanismos focales de los terremotos (Kaverina et al., 1996; Álvarez-Gómez 2014, 2019; Pérez-López et al., 2020), que permite agruparlos en función de la tipología de falla/mecanismo focal: desgarre, inverso, normal o combinado. Sobre una distribución bidimensional del k', hemos realizado una serie de perfiles en profundidad transversales a la Fosa Mesoamericana en relación a la geometría de la subducción, interpolando también los valores de k' en profundidad, lo que permite reconstruir el estado de deformación 3D.

El análisis 3D de la deformación muestra la distribución espacial homogénea de regímenes tectónicos extensionales, compresionales y en desgarre, obtenidos a partir de los diagramas de k'. La ventaja de este análisis frente al estudio de los campos de esfuerzos es que permite realizar un estudio cinemático de la deformación en relación con las principales estructuras tectónicas de la zona, por lo que permite correlacionar el comportamiento mecánico y la geometría de dichas estructuras, y además complementa otros análisis a partir del estudio de terremotos, como por ejemplo los estudios de campos de esfuerzos activos en la zona (e.g. Suter et al., 1991; Singh y Pardo, 1993), la tomografía sísmica (e.g. Husker y Davis, 2009), o los estudios de la partición de las ondas sísmicas transversales (shear wave splitting, e.g. Valenzuela y León-Soto, 2017).

El área de trabajo comprende la zona de convergencia de la Fosa Mesoamericana (FMA) en toda su zona de influencia mexicana y sus sectores próximos (Figura 1): la placa Pacífica (PA), 
la placa Rivera (RIV), la placa Norteamericana (NAM) y la placa de Cocos (CG), así como de sus principales límites de placa (e.g. Pardo y Suárez, 1993). Para llevar a cabo este trabajo, se han utilizado más de 1300 terremotos con solución del mecanismo focal obtenidos del catálogo Global Centroid-Moment-Tensor (CMT; https://www. globalcmt.org, último acceso julio 2020), y llevado a cabo el análisis por provincias: (a) en la zona de desgarre del Golfo de California, (b) la FMA, (c) la zona de convergencia de la placa RIV y la zona de la dorsal pacífica, (d) así como la terminación SE de la FMA (Figura 1). La ventaja de utilizar esta base de datos es que incorpora los parámetros de calidad de la base estadounidense del NEIC (National Earthquake Information Center, http://neic. usgs.gov/neis/epic/epic_global.html) del USGS, así como las mejoras en el cálculo de las magnitudes de momento para terremotos superiores a $\mathrm{M}$ 5 y de su mecanismo focal, reduciendo sus incertidumbres y asegurando una homogeneidad en su base de datos y completitud para $\mathrm{M} \geq 5$ desde su origen (Ekström et al., 2012). Además, la escala de trabajo del área de estudio, la cual presenta una extensión definida por una longitud superior a $4000 \mathrm{~km}$ y un área cercana a 7 x $10^{6} \mathrm{~km}^{2}$, implica que los estudios de agrupamientos de terremotos, tanto a nivel epicentral como hipocentral diluyan

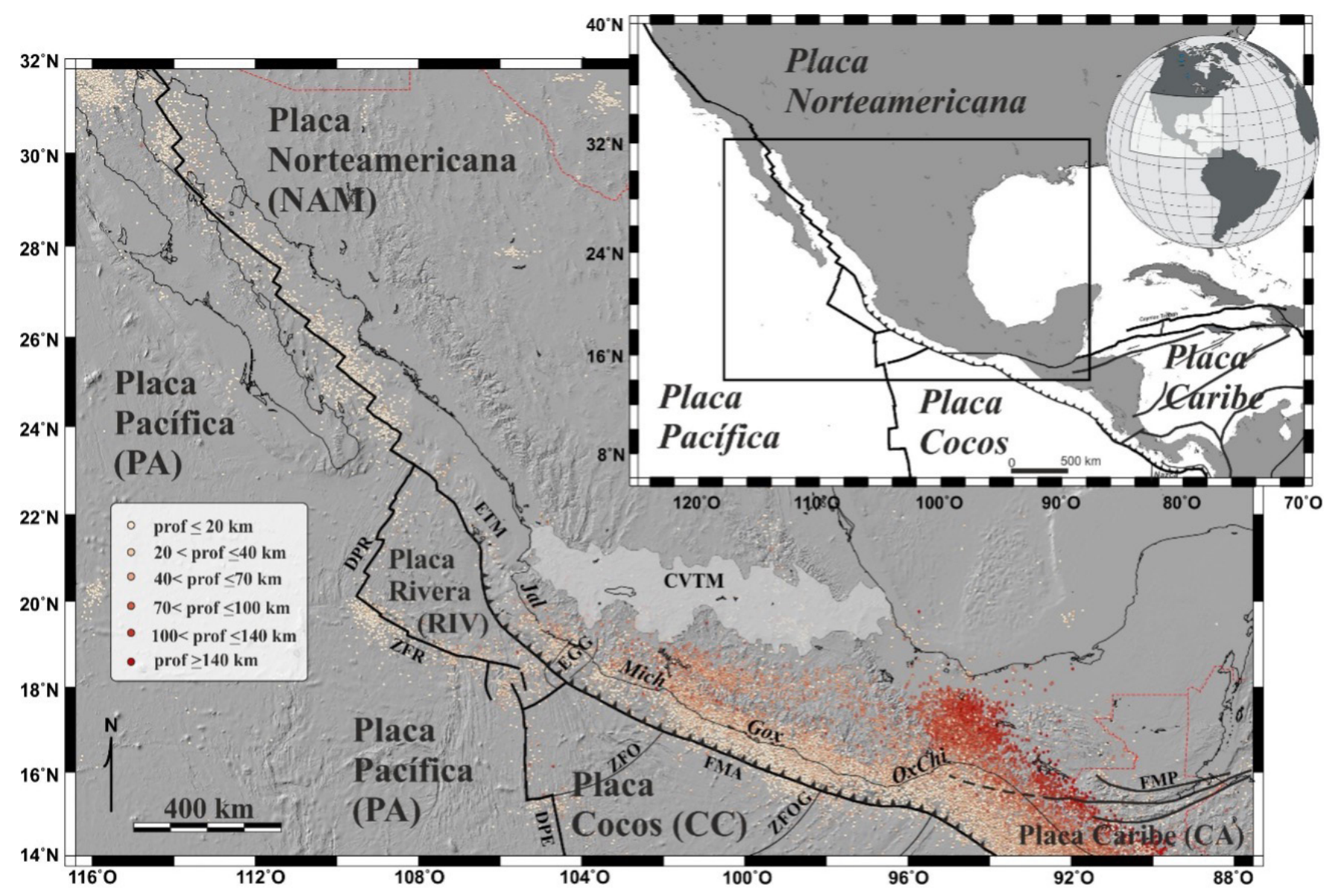

Figura 1 a) Situación de la zona de análisis. b) Esquema tectónico y localización de los epicentros de terremotos localizados en el área considerada (Iris Earthquake browser: http://ds.iris.edu/ieb/, último acceso agosto 2020). CVTM Cinturón Volcánico Transmexicano, ZFR Zona de falla de Rivera. ZFO Zona de Falla de Orozco, ZFOG Zona de Falla de O Gorman, DPR Dorsal Pacífico Rivera, DPE Dorsal Pacífico Este, FMA Fosa Mesoamericana, FMP Zona de Falla de Motagua Polochic, EGG El Gordo Graben, ETM Escarpe Tres Marías. Zonas: Jal: Jalisco; Mich: Michoacán, GOX: Guerrero - Oaxaca, OxChi: Oaxaca Chiapas. 
el error de localización, máxime si se tiene en cuenta que en este trabajo se han utilizado más de 1300 mecanismo focales. Obviamente, la completitud y el error de los catálogos sísmicos varía en el tiempo, en función de la cobertura de redes sísmicas, así como de los algoritmos y software de obtención del momento sísmico y de interpretación de los sismogramas (Kagan, 2003; Ekström et al., 2012). En cualquier caso, Kagan (2003) señala que los errores en orientación de los planos nodales de las soluciones del CMT entre el periodo $1977-2002$ son entre $5^{\circ}$ y $7^{\circ}$ para una magnitud de completitud de M 5.5, valor que ha sido disminuido para M 5 e incluso menor (M 4.8), según señala Ekström et al., (2012) hasta esa fecha.

Los resultados obtenidos en este trabajo presentan un modelo cinemático de la FMA donde se determinan tanto aquellas zonas donde aparecen permutaciones en el eje de máximo acortamiento horizontal $\left(\mathrm{e}_{\mathrm{y}}\right)$, generalmente en zonas cercanas a la zona de la FMA y paralelo a la misma (NNO-SSE), como los cambios en el tensor de deformación que pueden zonificar la subducción CG-NAM.

\section{Marco tectónico de la FMA}

Las placas oceánicas de Cocos (CG) y Rivera (RIV) subducen hacia el ENE bajo la placa norteamericana (NAM), y más al sureste a la Caribe (CA), a lo largo de la Fosa Mesoamericana (FMA, Figura 1). Esta fosa presenta una geometría tridimensional compleja con grandes variaciones del ángulo de subducción, así como un acoplamiento mecánico variable en función de la profundidad y del ángulo de la subducción (Suter, 1991; Singh and Pardo, 1993; Pardo y Suárez, 1993; 1995; Gardi et al., 2000; Guzmán-Speziale y Gómez-González, 2006; Yang et al., 2009; Manea y Manea, 2011; Kim et al., 2012; Corbo-Camargo et al., 2013; Álvarez y Yutsis, 2015; Fasola et al., 2016; Guzmán-Speziale y Zúñiga, 2016; Manea et al., 2017; Letort et al., 2018; Rodríguez-Pérez et al., 2020). Toda esta complejidad geométrica es la responsable de una distribución espacial y temporal de grandes terremotos inversos en la FMA, con la existencia de la laguna sísmica ( $g a p$ ) de Guerrero (Suárez et al., 1990; Kostoglodov et al., 1996, 2003), así como de terremotos de magnitud superior a $\mathrm{M}$ 5 y M 6 en falla normal (Gardi et al., 2000), y de terremotos destructivos de intraplaca en la zona central de magnitud M 7 (Langridge et al., 2000; Rodríguez-Pascua et al., 2017).

Además, el comportamiento del flujo mantélico en relación a su posición con respecto a la subducción (parte superior o inferior), complica un poco más el esquema, siendo el responsable último de la distribución espacial del vulcanismo tras-arco que aparece desde el Cenozoico en el centro de México (Yang et al., 2009; León-Soto et al., 2009).

\subsection{GEOMETRÍA Y ZONACIÓN DE LA FMA}

La Fosa Mesoamericana (FMA) presenta una geometría variable de $\mathrm{NO}$ a $\mathrm{SE}$ en función de la interacción entre las diferentes placas tectónicas que configuran este límite de placa (Pardo y Suárez, 1995). El ángulo de subducción en la zona central de la FMA es sub-horizontal, aumentando el buzamiento en el límite CC-CA hacia el SE y en el límite RIV-NAM hacia el NO. Este cambio de buzamiento se explica por el desacoplamiento entre la placa subducida y subducente en cada zona. Pardo y Suárez (1995) definen a partir del estudio de la sismicidad instrumental y de los mecanismos focales, cuatro regiones principales (Figura 1): (1) Región de Jalisco (Jal) definida por los límites RIV-NAM; (2) Región de Michoacán (Mich), donde decrece el ángulo de subducción según avanza hacia el sureste; (3) Región de Guerrero-Oaxaca (GOx), limitada por la zona de Falla de Orozco y la de O'Gorman donde la subducción presenta un ángulo sub-horizontal y, finalmente, (4) la Región de Oaxaca-Chiapas (OxChi), donde aumenta progresivamente el ángulo de subducción según avanzamos hacia la parte centroamericana. Esta geometría compleja con cambios laterales del ángulo de subducción en forma de 
rampas y rellanos, sería la responsable de que el Cinturón Volcánico Transmexicano (CVTM) no sea paralelo a la orientación de la FMA (Pardo y Suárez, 1995), mostrando una distribución lateral de diferentes estilos de volcanismo (Ferrari, 2004; Gómez-Tuena et al., 2007; Ferrari et al., 2012; Gómez-Tuena et al., 2018), así como la existencia del mayor campo de vulcanismo monogenético asociado a una cuenca tras-arco (Hasenaka, 1994).

La subducción de las placas RIV y CG, es una subducción con cambio de buzamiento a $40 \mathrm{~km}$ de profundidad, presentando un cinturón volcánico paralelo a la fosa, siendo el volcán Colima el más emblemático y con un volcanismo asociado de tipo calcoalcalino (Pardo y Suárez, 1993). El límite oeste de la placa RIV con la PA aparece segmentado en echelón, a partir de una rotación de $15^{\circ}$ durante el Pleistoceno con la génesis de terremotos principalmente en desgarre (Lonsdale, 1995).

Álvarez y Yutsis (2015) proponen un modelo tectónico con un límite neto entre las placas de RIV y CG en el Complejo Volcánico de Colima, con una orientación aproximada según NNE-SSO perpendicular a la FMA. Para ello, realizan varios perfiles gravimétricos sobre la cartografía de la anomalía de Bouger, tanto en la zona marina, como en la zona continental mexicana. Estos autores proponen un modelo de transpresión en la zona de subducción RIV-NAM, y de transtensión en la zona de subducción CG y CA, siendo el Complejo Volcánico de Colima la zona de unión entre ambas, con un movimiento relativo entre las placas de CG y RIV entre 2 y 3 cm/año (DeMets y Wilson, 1997). En su modelo, estos autores incluyen el modelo de flujo mantélico toroidal de Yang et al. (2009). En el modelo original de estos autores, se describe la geometría de la subducción de las placas RIV y CG a partir de los $150 \mathrm{~km}$ de profundidad, y proponen el Rift de Colima como nexo de unión entre ambas placas, con un aumento en los ángulos de subducción, disponiéndose sub-verticales a partir de los $100 \mathrm{~km}$ de profundidad. Además, estos autores sugieren que la relación actual entre la subducción de la placa RIV y el CVTM a 140 $\mathrm{km}$ de profundidad es geométricamente anómala, posiblemente debido a la presencia de un bloque remanente de subducción de la placa RIV bajo el CVTM, lo que genera una geometría en roll-over en la placa subducente (RIV) (Yang et al., 2009). Gardi et al. (2000) proponen un modelo sobre la distribución y presencia de grandes terremotos con componente normal en la zona de subducción de la FMA, dentro de la región Mich (región 2), zona central de México (Figura 1). Su modelo se basa en que estos terremotos están localizados en la zona inferior de la subducción, en la zona de interfase que está fuertemente acoplada. Estos autores explican la ocurrencia de dichos terremotos a partir de la flexura de la lámina subducente asociada a su geometría, frente a un mecanismo de subducción de tipo pulling-slab (Gardi et al., 2000). Según su modelo, no es necesario un cambio de estado de esfuerzos que explique la aparición de grandes terremotos inversos con terremotos normales precedentes, dentro del ciclo sísmico del segmento de la FMA de la región de Michoacán (región 2, Figura 1). Los elevados valores de deformación de la convergencia entre las dos placas (decenas de cm/año), generan zonas extensionales persistentes por debajo de la lámina de subducción en la región sismogénica, mientras que las grandes caídas de esfuerzos en la zona tienden a invertir dicho comportamiento extensional, generando así los mega terremotos de subducción (Gardi et al., 2000).

En cuanto a la geometría de unión del punto triple de la placa de CG con CA y NAM, Álvarez-Gómez et al. (2019) establecen un modelo cinemático de pull and push donde el modelo de arrastre de la lámina de subducción se adapta mejor a las observaciones GPS y de los terremotos en la zona tras-arco.

\subsection{ESTADO DE ESFUERZOS DE LA FMA}

El primer trabajo que muestra de forma global la distribución de esfuerzos en la FMA y en México en su conjunto es el de Suter (1991), donde a partir de datos de mecanismos focales hasta esa fecha (1991), datos de estría de falla y análisis de breakout en sondeos, caracteriza diferentes "provincias de 


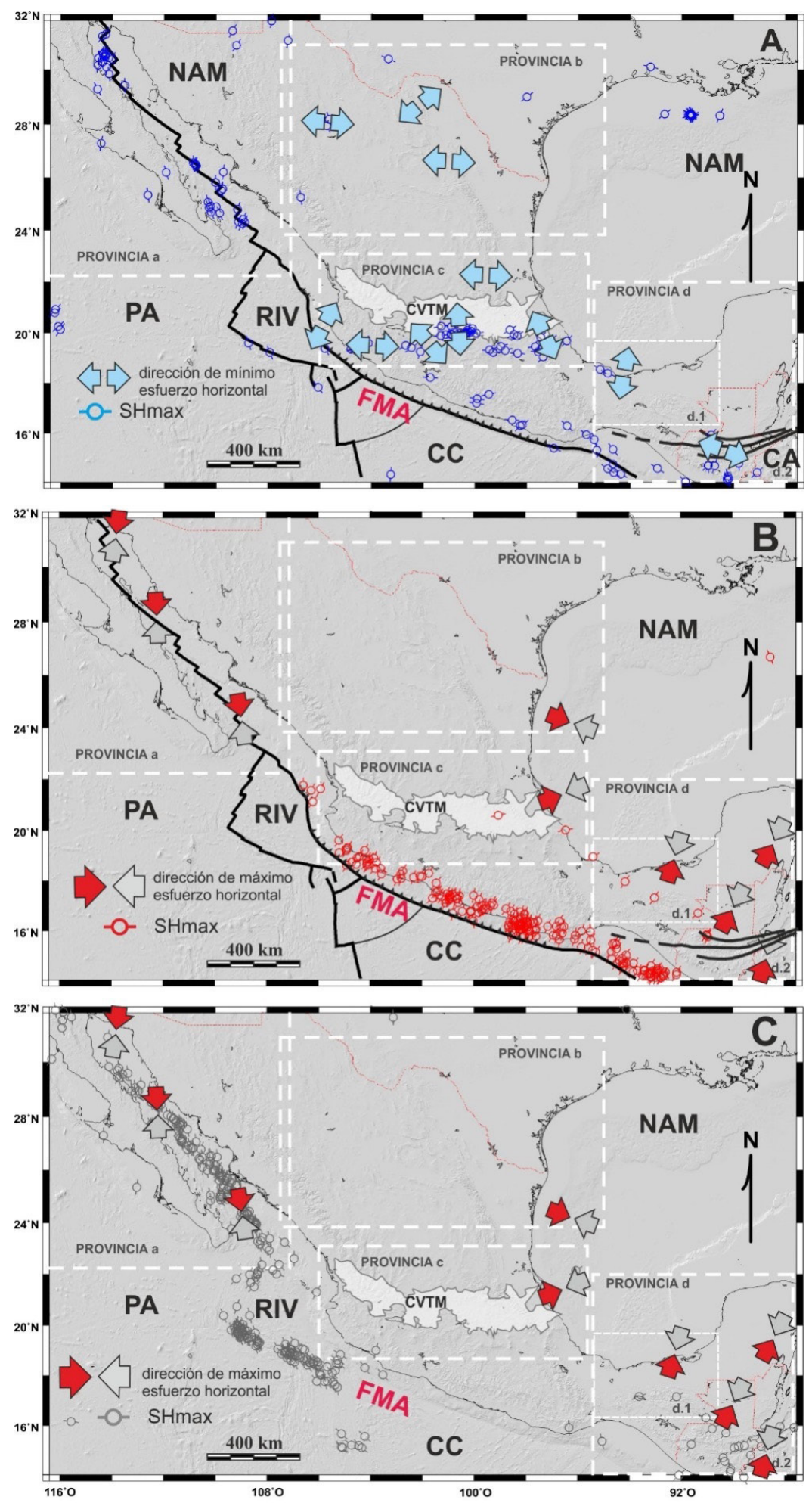

Figura 2 Mapa de provincias de esfuerzos descritas por Suter (1991), en relación a las principales placas tectónicas de la Fosa Mesoamericana (FMA). PA: Placa Pacífica; NAM: Placa Norteamericana; CC: Placa de Cocos y RIV: Placa Rivera. Las flechas hacen referencia a la orientación de $S_{H \max }$ (máximo esfuerzo horizontal, flecha roja y gris) y $\mathrm{S}_{\mathrm{hmin}}$ (mínimo esfuerzo horizontal, flecha azul), Suter, (1991). Datos de esfuerzos $\left(S_{\mathrm{H} \max }\right.$ ) de World Stress Map (Heidbach et al., 2016a, 2016b) separados por regímenes tectónicos: A. Régimen Tectónico Extensional; B. Régimen Tectónico Compresivo, la flechas rojas y grises hacen referencia a la dirección de $\mathrm{S}_{\mathrm{Hmax}}$ compresivo y en desgarre, según los datos de Suter (1991); C. Régimen Tectónico en Desgarre. 
esfuerzos" (stress provinces), en función de si el tensor es de tipo desgarre, normal o inverso (Figura 2). Las provincias definidas son: (a) la zona NO de México (Golfo de California, Península de Baja California y la parte correspondiente central de México) caracterizada por fallas normales y de rumbo. En esta zona define $\mathrm{S}_{\mathrm{Hmax}}$ (esfuerzo máximo en la horizontal) según NNO; (b) provincia NE de México definida como una zona de baja sismicidad intraplaca, y con una orientación de $\mathrm{S}_{\text {Hmax }}$ variable de E-O a NO-SE, con fallas activas N-S y NNE-SSO; (c) provincia central de México, caracterizada por el Cinturón Volcánico Transmexicano, con un campo de esfuerzos extensional $\left(\mathrm{S}_{\mathrm{Hmax}}\right.$ según ENE) con fallas normales orientadas E-O; (d) provincia sur de México y zona oeste de América Central con dos sub-provincias: (d.1) con un $\mathrm{S}_{\text {Hmax }}$ NNE paralelo al margen continental en Golfo de México, Veracruz y Tabasco, caracterizado por la presencia tanto de fallas normales como de fallas inversas. Ambos conjuntos de fallas se explican mediante un intercambio de ejes de esfuerzos en el dominio convergente y el dominio extensional de esta subprovincia; y finalmente (d.2) con un tensor de esfuerzos en desgarre con $\mathrm{S}_{\text {Hmax }}$ según NE-SO.

En cuanto al mapa de esfuerzos en la zona de estudio a partir de los datos del World Stress Map (WSM16, Heidbach et al., 2016a, 2016b, último acceso el 1 de septiembre, 2020), la figura 2 muestra los datos separados por regímenes tectónicos extensionales (200 datos; Figura 2A), compresivos (298 datos; Figura 2B) y en desgarre (598; Figura 2C). En total, 1096 datos con tensor asignado, que corresponden a mecanismos focales, sondeos, y otros datos geológicos (i.e. datos de estría sobre plano de falla activa).

En cuanto al régimen de esfuerzos de tipo extensional (Figura 2A) aparece principalmente localizado en el CVTM, en la provincia (c) de Suter (1991), así como en su provincia (a) a lo largo del límite de placa PA con NAM, en la zona NO de México. Se observa una amplia distribución de terremotos con mecanismo focal de tipo normal, que estarían relacionados con el acoplamiento de la lámina de subducción en la FMA (Gardi et al.,
2000), y con un régimen transtensivo propio del límite de placa PA-NAM. El campo de esfuerzos compresivo (Figura 2B) se circunscribe principalmente a la FMA y a los primeros $50 \mathrm{~km}$ de profundidad de la litosfera (Petricca y Carminati, 2016). En la convergencia de RIV-NAM, la geometría variable de la lámina de subducción hace que sea una zona más compleja, con escasos datos de esfuerzos convergentes; mientras que el límite de placas RIV-PA es de tipo desgarre (dorsal PA - RIV y zona de Falla de Rivera, ZFR, Figuras 1, 2C), aunque la mayoría de la deformación de este tipo se concentra en la provincia (a), en el Golfo de California, en la zona NO de México (Suter, 1991; Figura 2C). En el análisis que hemos llevado a cabo en este trabajo, se han utilizado los datos de los mecanismos focales obtenidos de la base de datos CMT (https://www.globalcmt.org, último acceso 25 junio 2020), los cuales se integrarán con los datos de WSM16 para el estudio conjunto de los diferentes regímenes tectónicos a lo largo de la FMA, así como las localizaciones hipocentrales de la base de datos IRIS (Iris Earthquake browser: http://ds.iris.edu/ieb/, último acceso agosto 2020).

\subsection{MOVIMIENTO RELATIVO ENTRE LAS PLAGAS TEGTÓNICAS}

La velocidad relativa de movimiento entre las placas de RIV, CG, PA, CA y NAM ha sido estudiada por diversos autores a partir de datos de GPS, estudio de los terremotos y de la distribución espacial de la anomalía magnética en la zona de la Dorsal Pacífico-Rivera (DeMets et al., 1990; Suárez et al., 2003; Guzmán-Speziale y Gómez-González, 2006; DeMets et al., 2010; Peláez-Gaviria et al., 2013, entre otros). En la zona de convergencia entre las placas de RIV y NAM, la velocidad oscila entre 9 y $43 \mathrm{~mm} / \mathrm{a}$ (Suárez et al., 2003), mientras que hacia el SE va aumentando su valor de 48 a $72 \mathrm{~mm} / \mathrm{a}$ (Figura 3, DeMets et al., 1990). Guzmán-Speziale y Gómez-González (2006) calculan la tasa de deformación sísmica de la subducción entre las placas NAM-CG $\left(2.8 \times 10-7 \mathrm{yr}^{-1}\right)$ y CG-CA $(3.7$ $\left.\times 10-8 \mathrm{yr}^{-1}\right)$, utilizando los mecanismos focales 
de los terremotos inversos registrados en la zona durante casi 30 años. Ambos autores sugieren que en la zona de NAM-CG se acomoda un $80 \%$ de la deformación tectónica en forma de terremotos inversos, mientras que en la zona de CG-CA este valor no excede el 10\%. Dicho comportamiento sería consecuencia del cambio de geometría de la subducción y la incorporación del prisma trasarco (forearc-sliver) de la placa CA en la placa CC.

\section{Metodología}

La litosfera terrestre se encuentra en un estado permanente de deformación debido al estado de esfuerzos derivado de la tectónica de placas, la cual incluye multitud de orígenes para aquellos estados de deformación cortical que seamos capaces de determinar. La dinámica terrestre derivada de esta actividad tectónica es la responsable de la génesis de los campos de esfuerzo y deformación, los cuales a su vez son los responsables de la actividad de las fallas que disparan los terremotos. En este trabajo, se han utilizado los métodos de estudio del campo de deformación tectónica a partir de datos de los mecanismos focales de los terremotos registrados en la zona de estudio (González-Casado et al., 2000; Herraiz et al., 2000; Giner-Robles et al., 2003a, 2003b, 2008, 2009).

En este trabajo nos decantamos por la representación de los tensores de deformación a partir del estudio de los mecanismos focales mediante

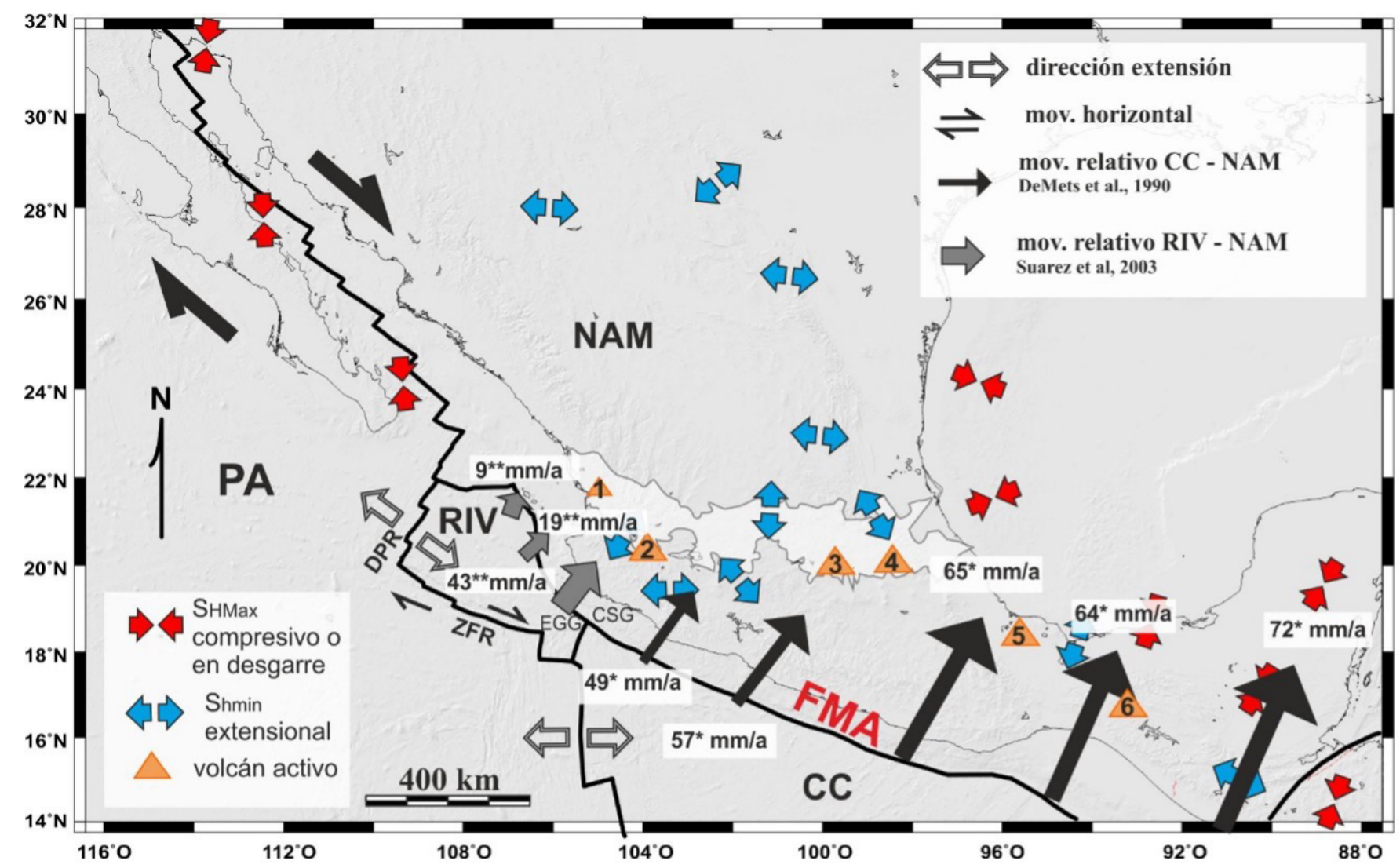

Figura 3 Velocidades relativas de las placas tectónicas en el área de estudio (* De Mets et al., 1990; ** Suárez et al., 2003). FMA: Fosa Mesoamericana; PA: Placa Pacífica; NAM: Placa Norteamericana; CC: Placa de Cocos y RIV: Placa Rivera. Las flechas hacen referencia a la orientación de $S_{\text {Hmax }}$ (máximo esfuerzo horizontal, flecha roja) y $S_{\text {hmin }}$ (mínimo esfuerzo horizontal, flecha azul) (Suter, 1991). Se incluyen los principales volcanes activos (triángulos naranjas), de izquierda a derecha: 1: Cerobuco, 2: Colima; 3: Popocatépetl; 4: Pico de Orizaba; 5: Volcán de San Martín y 6: El Chicón. ZFR: Zona de Fractura de Rivera; EGG: El Godo Graben, SCG: Sur Colima Graben. 
el Método de los Diedros Rectos y el Modelo de Deslizamiento, porque sus resultados representan la cinemática de la deformación tectónica, lo que permite cuantificar la geometría de la deformación y relacionarla con las estructuras tectónicas principales definidas en la zona de estudio. En este contexto, Marret y Peacock (1999) diferencian el uso de los tensores de esfuerzo/deformación tectónica en relación a los análisis de tipo dinámico (causa) y cinemático (efecto) respectivamente. La ventaja del uso de los tensores de deformación es que se ciñen a la observación y describen los patrones asociados a las rotaciones y traslaciones congruentes con el marco tectónico definido por las estructuras conocidas: la zona de subducción, fallas asociadas, zonas acopladas, etc. (Marret y Peacock, 1999). El análisis de la deformación que hemos realizado no pretende realizar modelizaciones matemáticas ni computaciones analíticas de los campos de esfuerzos.

Las técnicas de análisis de la deformación tectónica que se han utilizado son: (1) el Método de los Diedros Rectos (DR) (Angelier y Mechler, 1977) y (2) el Modelo de Deslizamiento (MD) (Reches, 1983; De Vicente, 1988). Del análisis con ambos métodos, podemos determinar la orientación de máximo acortamiento horizontal $\left(\mathrm{e}_{\mathrm{v}}\right)$, y la forma del tensor de deformación $\left(\mathrm{k}^{\mathrm{s}}\right)$, definido como la relación entre $e_{\mathrm{y}} \mathrm{y}$ el acortamiento vertical $\left(\mathrm{e}_{\mathrm{z}}\right)$. Ambas técnicas de análisis son robustas y han sido utilizadas ampliamente para la reconstrucción del tensor de deformación en diferentes contextos y a diferentes escalas aplicadas tanto en el análisis de mecanismos focales de terremotos (e.g. González Casado et al., 2000; Giner-Robles et al., 2003a, 2003b, 2009; Olaiz et al., 2009), como a poblaciones de fallas medidas en el terreno (e.g. De Vicente, 1987; Herraiz et al., 2000; Pérez-López et al., 2020).

Finalmente se establece el análisis cluster para determinar las variaciones 2D del estado de la deformación que, junto con el análisis de los perfiles en profundidad de los mecanismos focales, permite establecer el modelo 3D o tomografía de la deformación del complejo tectónico de las placas de RIV y CG subduciendo bajo la Placa NAM y CA. Estos análisis se han realizado mediante el agrupamiento por tensores compatibles (según el k', tipo de mecanismo/falla). Para ello utilizamos una combinación entre los agrupamientos del diagrama de Kaverina modificado por zonas, junto con el análisis del k’ del tensor de deformación.

\subsection{MÉTODOS DE ANÁLISIS DE LA DEFORMACIÓN}

El primer tipo de análisis, DR (Angelier y Mechler, 1977), consiste en un método cualitativo basado en la determinación geométrica de los cuatro cuadrantes generados por los dos planos nodales en cada uno de los mecanismos focales analizados. Dos de los cuadrantes indican compresión, mientras que los otros indican extensión. Para representar los resultados utilizamos el diagrama de diedros rectos que representa, en proyección estereográfica, las áreas comunes de extensión y compresión para un conjunto de mecanismos focales. La geometría resultante del diagrama permite establecer de forma cualitativa la orientación de máximo acortamiento horizontal $\left(\mathrm{e}_{\mathrm{y}}\right)$ y las tipologías de fallas predominantes en la población analizada (De Vicente et al., 1992). La principal ventaja de esta metodología es que el diagrama de diedros rectos resultante es independiente de que plano nodal corresponde a la falla real responsable del terremoto (Giner-Robles et al., 2006).

El segundo método, el MD (Reches, 1983; De Vicente, 1988) es un método cuantitativo que permite determinar el plano de falla responsable del terremoto (Capote et al., 1991) a partir de los planos nodales del mecanismo focal y que además, también proporciona información sobre la orientación y forma del tensor de deformación: (a) orientación de máximo acortamiento horizontal $\left(e_{\mathrm{y}}\right)$ y (b) el factor de forma del tensor ( $\left.\mathrm{k}^{\prime}\right)$, siendo k' $=\mathrm{e}_{\mathrm{y}} / \mathrm{e}_{\mathrm{z}}, \mathrm{y}$ con un rango de valores que oscila entre $-\infty \leq \mathrm{k}^{\prime} \leq+\infty$ (e.g. De Vicente, 1987; Pérez-López et al., 2020). El Modelo de Deslizamiento permite asignar un carácter teórico normal o inverso a cada plano de falla en función de los valores de buzamiento de la falla y el ángulo de cabeceo (pitch) de la estría sobre el plano de falla (De Vicente et al., 1988). La metodología propuesta por Capote et al. 
(1991) se basa en esta asignación teórica para discriminar cuál de los dos planos nodales es el plano de falla: el plano nodal cuyo carácter coincida con el definido para el mecanismo focal (en función de la posición de los ejes $\mathrm{P}, \mathrm{T}$ y B), es el plano de falla. Esta metodología tiene sus limitaciones en el análisis individual de un solo mecanismo ya que, según el Modelo de Deslizamiento, el plano seleccionado siempre correspondería mecánicamente a un plano neoformado frente al tensor de deformación definido.

No obstante, en este trabajo no pretendemos realizar un análisis detallado de un mecanismo focal individual, sino de un conjunto de mecanismos focales para obtener un tensor de deformación capaz de generar el conjunto analizado. En este tipo de análisis, la incertidumbre inherente a la utilización de esta metodología se minimiza, tal y como demuestran Giner-Robles et al. (2006), analizando 840 mecanismos focales en 9 zonas distintas repartidas por diferentes límites de placas (3 zonas de dorsal oceánica, 3 zonas de subducción y 3 zonas de fallas de desgarre o transformantes). Estos autores comparan los resultados obtenidos utilizando diferentes tipos de metodologías de análisis de la deformación y del esfuerzo, y diferentes criterios de selección del plano de falla (incluyendo el utilizado en este trabajo), concluyendo que el criterio de selección del plano de falla no influye significativamente en la determinación de tensores de deformación y esfuerzos en grandes poblaciones de datos.

La principal ventaja de este método es que podemos obtener, para cada mecanismo focal, la forma y la orientación del tensor de deformación asociado a la localización epicentral, orientación que se define como una elipse proyectada en el plano horizontal (donde $e_{x} y_{y} e_{y}$ son los ejes horizontales de la elipse de deformación, y e e eje vertical).

\subsection{GLASIFICACIÓN DE MEGANISMOS FOGALES}

El MD permite establecer la tipología de la falla en relación con el valor k' obtenido para cada uno de los mecanismos (Capote et al., 1991; De Vicente, 1988), definiendo las siguientes categorías: (a) falla normal pura, (b) falla normal transcurrente, (c) falla transcurrente normal, (d) falla transcurrente pura, (e) falla transcurrente inversa, (f) falla inversa transcurrente y $(\mathrm{g})$ falla inversa pura.

Para representar la tipología de las fallas/ mecanismos se ha utilizado el programa FMC desarrollado por Álvarez-Gómez (2014, 2019) basado en el trabajo de Kaverina et al. (1996), y que mejora el diagrama ternario propuesto por Frohlich y Apperson (1992) para la clasificación de mecanismos focales. En el trabajo original, el diagrama ternario clasifica cada mecanismo focal con base en el ángulo de inmersión (plunge o dip-angle) de los ejes P, T y B, definiendo siete posibles categorías (Figura 4a): (a) normal, (b) normal oblicuo, (c) desgarre normal, (d) desgarre puro, (e) desgarre inverso, (f) inverso oblicuo y (g) inverso.

En este trabajo hemos modificado el diagrama ternario resultante de acuerdo con los valores de la forma del tensor de deformación ( $\left.k^{\prime}\right)$ para clasificar las fallas / mecanismos, de forma similar a lo propuesto por Pérez-López et al. (2020). Esta modificación es similar a la clasificación original propuesta por Kaverina et al. (1996), ya que el MD también clasifica los mecanismos de forma geométrica, calculando el valor de $\mathrm{k}^{\prime}$ a partir de los valores y relación entre los ángulos de buzamiento y cabeceo de la falla seleccionada entre los dos planos nodales del mecanismo focal (Capote et al., 1991; De Vicente, 1987; Pérez-López et al., 2020) (Figura 4b). Si bien las zonas de distribución de las diferentes categorías varían ligeramente, la clasificación de mecanismos es muy similar a la definida por Kaverina et al. (1996) y de esta forma podemos representar las tipologías de fallas/ mecanismos siguiendo la clasificación propuesta en el MD utilizado en este trabajo (Figura 4).

La definición del factor de forma del tensor de deformación ( $\left.k^{\prime}\right)$ de cada uno de los mecanismos permite construir mapas de k' (Giner et al., 2008; Olaiz et al., 2009) a partir de la interpolación de los datos individuales del valor de $\mathrm{k}^{\prime}$ asignado a cada localización epicentral del terremoto. Además, 

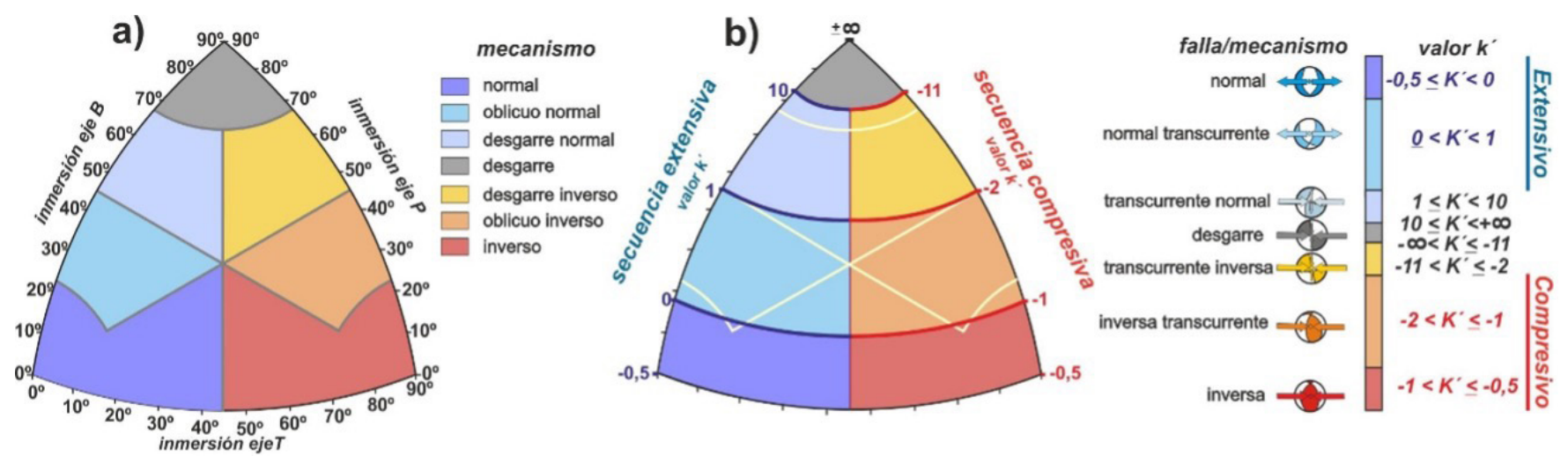

Figura 4 a) Diagrama ternario de clasificación de mecanismos focales propuesto por Kaverina et al. (1996) (salida gráfica del programa FMC, Álvarez Gómez, 2014, 2019). b) Diagrama ternario utilizado en este trabajo para la clasificación de fallas y mecanismos focales. Se utiliza el mismo tipo de representación, pero se modifican los campos de clasificación en función del parámetro de factor de forma del tensor de deformación ( $\left.k^{\prime}\right)$ propuesto por De Vicente $(1987,1988)$.

también es posible interpolar los valores de k' en profundidad para cortes seriados y perpendiculares a la Fosa Mesoamericana desde el NO hacia el SE, permitiendo realizar un análisis cluster 3D.

\subsection{ANÁLISIS “GLUSTER” DE LA DISTRIBUGIÓN ESPACIAL}

En zonas tectónicamente complejas, las variaciones locales en el tensor de deformación regional pueden quedar enmascaradas cuando se lleva a cabo un análisis conjunto de mecanismos focales (Giner-Robles et al., 2009). En este trabajo se ha realizado un análisis cluster de los mecanismos focales en función de los resultados individuales obtenidos de la aplicación del MD (González-Casado et al., 2000; Giner-Robles et al., 2003a, 2006, 2009).

En primer lugar, se ha clasificado cada solución de mecanismo focal individual utilizando el programa FMC modificado (Álvarez-Gómez, 2014, 2019). A continuación, se han analizado las construcciones de los mecanismos mediante el MD, obteniendo datos individuales de forma y orientación del tensor de deformación. Y finalmente se han agrupado espacialmente de acuerdo con los resultados individuales obtenidos en relación a la tipología de la falla y a la orientación y forma del tensor de deformación (mapas de $\left.\mathrm{k}^{\prime}\right)$. Estas agrupaciones de mecanismos han sido analizadas posteriormente con los dos métodos de análisis de la deformación propuestos (DR y MD).

Una vez analizados, se han construido las trayectorias de la deformación en función de los datos puntuales de e obtenidos del análisis individual, teniendo en cuenta las variaciones definidas en el análisis cluster (orientación de $\mathrm{e}_{\mathrm{v}}$ mapas de $\mathrm{k}^{\prime}$, etc.) de manera similar a la realizada por otros autores (González Casado et al., 2000; Herraiz et al., 2000; Giner-Robles et al., 2003a, 2009). La representación de las trayectorias de $\mathrm{e}_{\mathrm{y}} \mathrm{y} \mathrm{e}_{\mathrm{x}}$ nos permite discriminar claramente entre posibles permutaciones entre los ejes de deformación (misma orientación de trayectorias con intercambio de $\mathrm{e}_{\mathrm{y}}$ por $\mathrm{e}_{\mathrm{x}}$ ) y posibles cambios en la orientación producidos (cambios en la orientación de ambos ejes), por la presencia de grandes estructuras o límites (Giner-Robles et al., 2003b). Para la construcción de las trayectorias se ha utilizado el programa Lissage (Lee y Angelier, 1994), programa creado específicamente para la construcción de mapas de trayectorias a partir de datos locales.

Además, la realización de los perfiles en profundidad seriados a lo largo de la FMA con la interpolación de los valores de k', permite la reconstrucción del campo de deformación 3D y su relación con los principales límites de placas y fallas principales, así como la potencial existencia de acoplamientos, relaciones con flujos mantélicos. 


\section{Resultados}

Se han analizado más de 1300 terremotos con solución de mecanismo focal localizados en la zona de estudio, recopilados del catálogo Global Centroid Moment Tensor (CMT) (CMT, 2020). En este trabajo no se han introducido las incertidumbres de la fase $\mathrm{W}$ que incluyen el catálogo CMT, tal y como sugieren Duputel et al. (2012), al depender del organismo de cálculo la obtención de la covarianza y del modelo de Tierra utilizado. Valentine y Trampert (2012) también recalcan este punto, sobre todo en lo relativo al modelo de Tierra utilizado para obtener la inversión del momento sísmico. La ventaja del uso de la tomografía de la deformación es que al mejorar los datos de entrada y disminuir sus incertidumbres mejorará la distribución tridimensional de la deformación.

En cuanto a los errores en la estimación de los terremotos del catálogo de CMT, Ekström et al. (2012) presentan una notable mejora de los mismos, tanto en la estimación de la magnitud (terremotos con magnitud igual o superior a M 5) como en su localización epicentral. Por otro lado, la localización hipocentral se asume de la base de datos NEIC del USGS, siendo inferior a $10 \mathrm{~km}$ en cualquier caso. Debido a la escala de trabajo de este análisis, no es relevante el error asumido por la base CMT al no modificar de forma significativa la delimitación de las zonas de transición de tensores de deformación homogéneos. Consideramos esta asunción válida tanto para la distribución epicentral, como hipocentral.

En cuanto a la base de datos del programa Global CMT, las profundidades de los mecanismos varían entre 10 y $273 \mathrm{~km}$, aunque en general son terremotos superficiales $(50.2 \%$, prof. $\leq 20 \mathrm{~km}$; $25.2 \%, 20 \leq$ prof. $<40 \mathrm{~km} ; 11.5 \%, 40 \leq$ prof. $\leq 70$ $\mathrm{km} ; 6.0 \%, 70 \leq$ prof. $\leq 100 \mathrm{~km} ; 3.0 \%, 100 \leq$ prof. $\leq 140 \mathrm{~km} ; 4.1 \%$ prof. $>140 \mathrm{~km})$, concentrándose los terremotos con profundidades superiores a 70 $\mathrm{km}$ en la FMA. Las magnitudes de los terremotos aparecen homogeneizadas en la base de datos a magnitud de momento Mw, y varían entre 4.0 y 8.0.

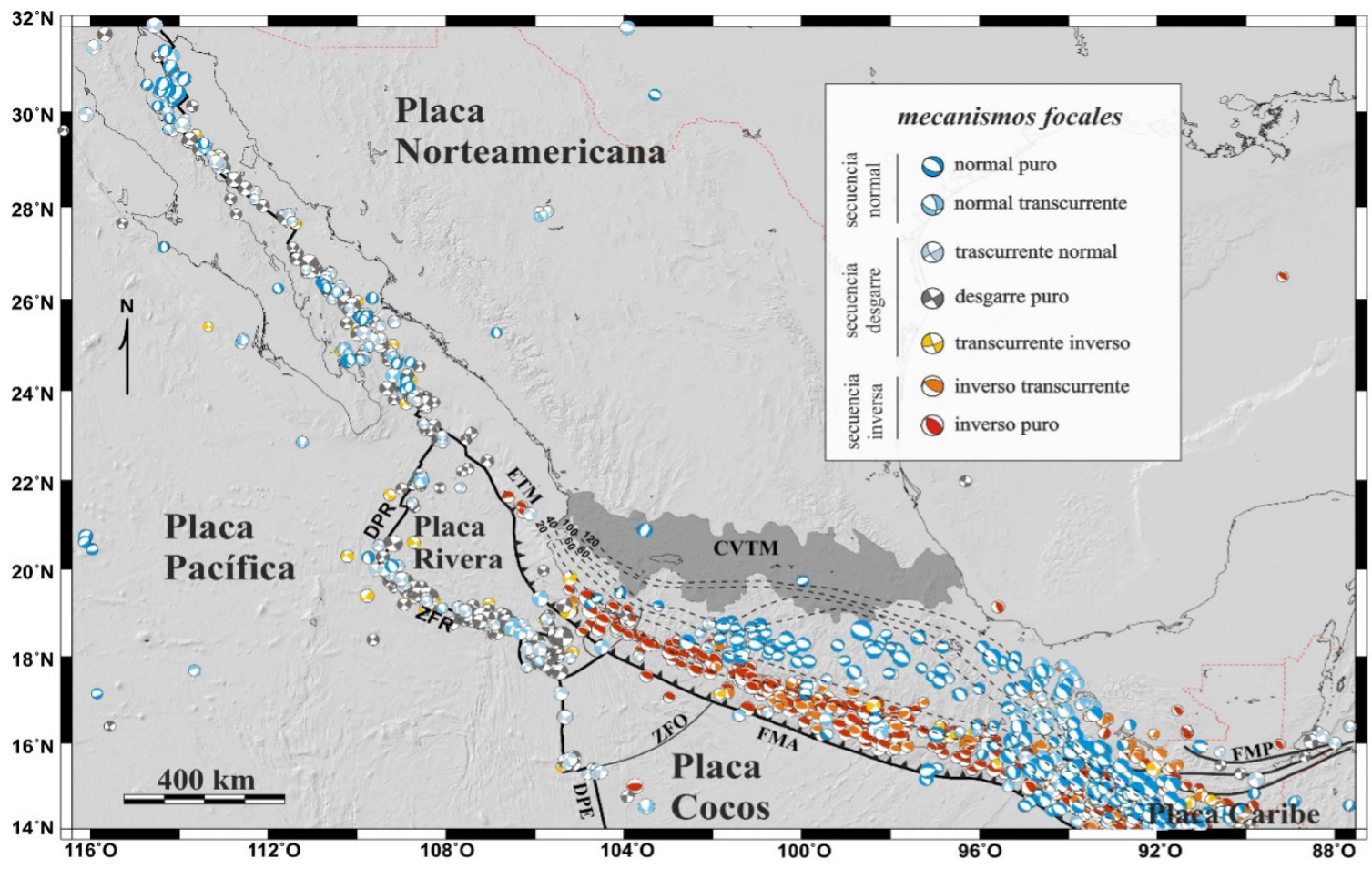

Figura 5 Localización y clasificación de los mecanismos focales situados en la zona de análisis. La clasificación se ha realizado utilizando el diagrama ternario modificado de Kaverina et al. (1996). Las líneas negras discontinuas representan las de la lámina de subducción (en km) (Pardo y Suárez, 1996). 
Se ha realizado un primer análisis clasificando los mecanismos en función de la tipología de falla/mecanismo mediante el diagrama ternario modificado de Kaverina et al. (1996) (ver Figura 4b): el $26.2 \%$ de los mecanismos pertenecen a la secuencia normal $(16.7 \%$ normales, $9.5 \%$ normal transcurrentes), el $35.5 \%$ a la secuencia de desgarre $(9.9 \%$ transcurrentes normales, $20.3 \%$ desagarres puros, $5.3 \%$ transcurrentes inversos), y el $38.3 \%$ restante a la secuencia inversa $(6 \%$ inverso transcurrentes, 32,3\% inversos) (Figura 5).

$\mathrm{Su}$ distribución con respecto a los grandes límites de placas (Figura 6) muestra variaciones importantes. En el límite de PA-NAM, el diagrama muestra una distribución claramente relacionada con el tipo de límite presente: zonas de dorsal y fallas transformantes (mecanismos de la serie extensiva y de desgarre) (Figura 6a). En cambio, los límites entre la Placa Pacífica y las Placas de Rivera (PA/RIV, Zona de Falla de Rivera y Dorsal Pacífico-Rivera) y de Cocos (PA/CG, Dorsal del
Pacífico-Este) (Figura 6b, c), siendo límites muy similares al límite PA/NAM, muestran casi exclusivamente mecanismos de la serie de desgarre.

El límite definido por la FMA (Figura 6) presenta resultados muy similares tanto en el límite de la Placa Norteamericana con las placas de Rivera (RIV/NAM) y de Cocos (CG/NAM), como en el límite entre las placas de Cocos y de Caribe (CG/ CA). Estos límites presentan mayoritariamente mecanismos de la serie inversa, aunque presentan un número importante de mecanismos de la serie normal, sobre todo hacia el SE de la zona analizada. No obstante, es importante señalar que el límite entre las placas Rivera y Norteamericana (RIV/NAM) (Figura 6d) aparece menos definido por la desaparición de la Fosa Mesoamericana hacia el NO. Finalmente, el límite entre la Placa Caribe y la Norteamericana (CA/NAM, Figura $6 \mathrm{~g})$, muestra una gran variación en la tipología de mecanismos, ya que presenta una combinación entre mecanismos propios de un límite compresivo

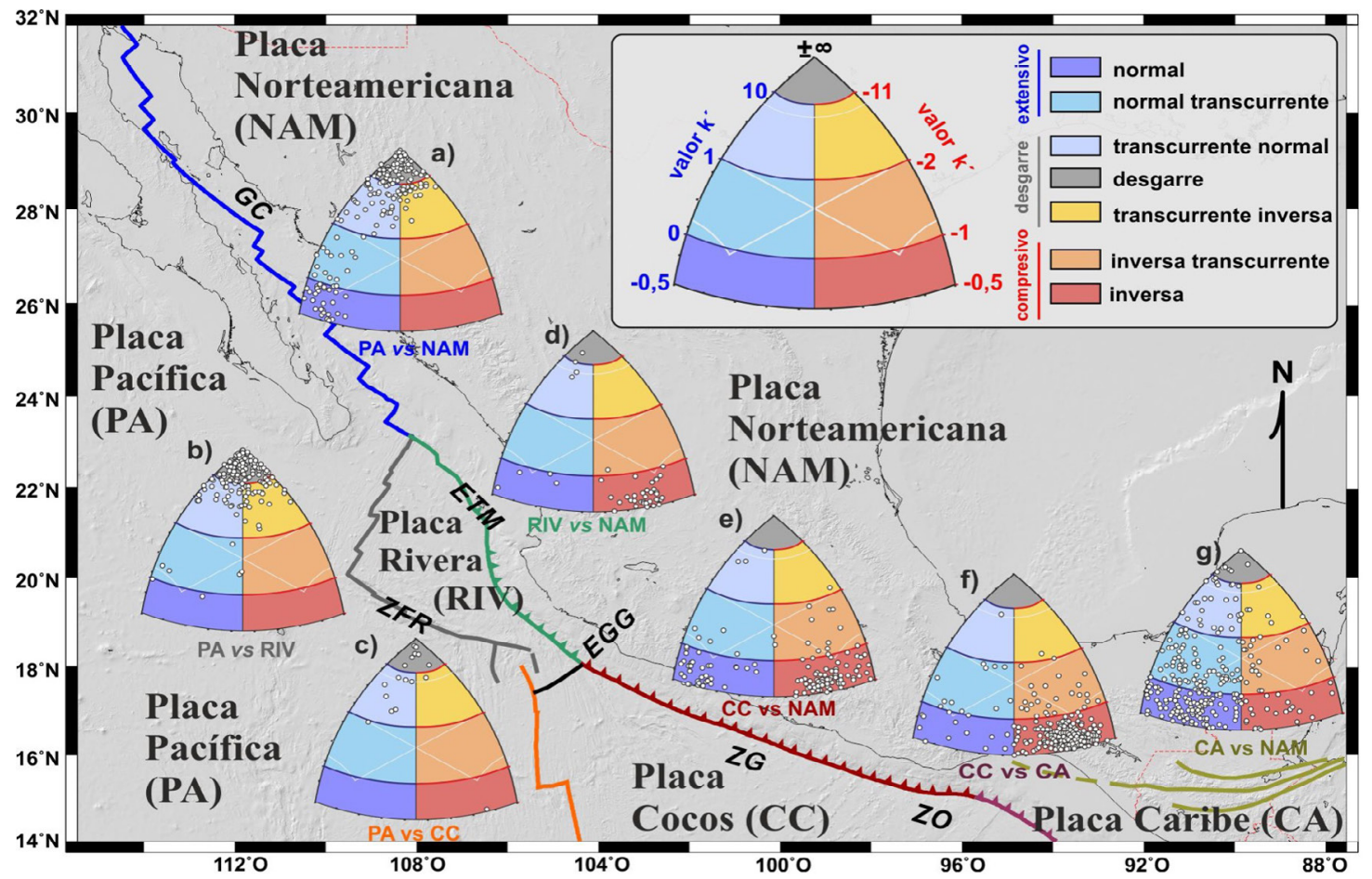

Figura 6 Diagramas ternarios de clasificación de los mecanismos focales (Kaverina et al.,1996) situados en los principales límites de placas de la zona: a) límite Placa Pacífica-Placa Norteamericana (en azul), b) límite Placa Pacífica-Placa de Rivera (en gris), c) límite Placa Pacífica-Placa de Cocos (en naranja), d) límite Placa de Rivera-Placa Norteamericana (en verde), e) límite Placa de Cocos-Placa Norteamericana (en rojo), f) límite Placa de Cocos-Placa Caribe (en morado), g) límite Placa Caribe-Placa Norteamericana (en amarillo). GC Golfo de California, ETM Escarpe Tres Marías, ZFR Zona de Falla de Rivera, EGG Graben de El Gordo, ZG Zona de Guerrero, ZO Zona de Oaxaca. 
y la de mecanismos propios de un límite por fallas de desgarre (Sistema de Fallas Motagua-Polochic), mostrando la complejidad de este límite.

$\mathrm{El}$ análisis individual de los mecanismos focales mediante el MD nos proporciona datos puntuales de orientación del tensor de deformación en la horizontal (Figura 7), mostrando claramente las direcciones de acortamiento en la FMA en el rumbo NE-SO. Los datos puntuales del factor de forma del tensor de deformación ( $\left.k^{\prime}\right)$ están representados por el mapa interpolado de los datos individuales de $\mathrm{k}^{\prime}$.

La zona del Golfo de California (Figuras 6a y 8) (zona 1) presenta datos muy homogéneos en relación al tipo de falla, mostrando fundamentalmente terremotos de falla de desgarre y normales, mostrando claramente un límite dorsal-transformante. Se han establecido 4 zonas en función de la distribución espacial de los mecanismos focales y su relación con las principales estructuras que definen este límite que presentan resultados muy similares en el análisis de la deformación.

Por otro lado, se ha realizado la zonificación de la zona de confluencia de las Placas de Rivera, Cocos y Caribe con la Placa Norteamericana, a partir de la distribución espacial de los mecanismos focales con respecto a los principales límites de placas (Fosa Mesoamericana, Dorsal Pacífico-Rivera y Zona de Falla de Rivera), de su tipología (diagrama de Kaverina, ver Figura 6), y de las orientaciones individuales de $\mathrm{e}_{\mathrm{y}}$ obtenidas del análisis mediante el MD (zonas 2, 3, 4 y 5).

Se han establecido 15 sub-zonas de análisis (Figura 9) a partir de las 4 zonas principales en relación al tensor de deformación: zona 2a (Dorsal Pacífico-Rivera); zona 2b y 2c (Zona de Falla de Rivera); zona 2d (zona SE del límite PA-RIV); zona 3a (Escarpe Tres Marías); zona 3e (Dorsal

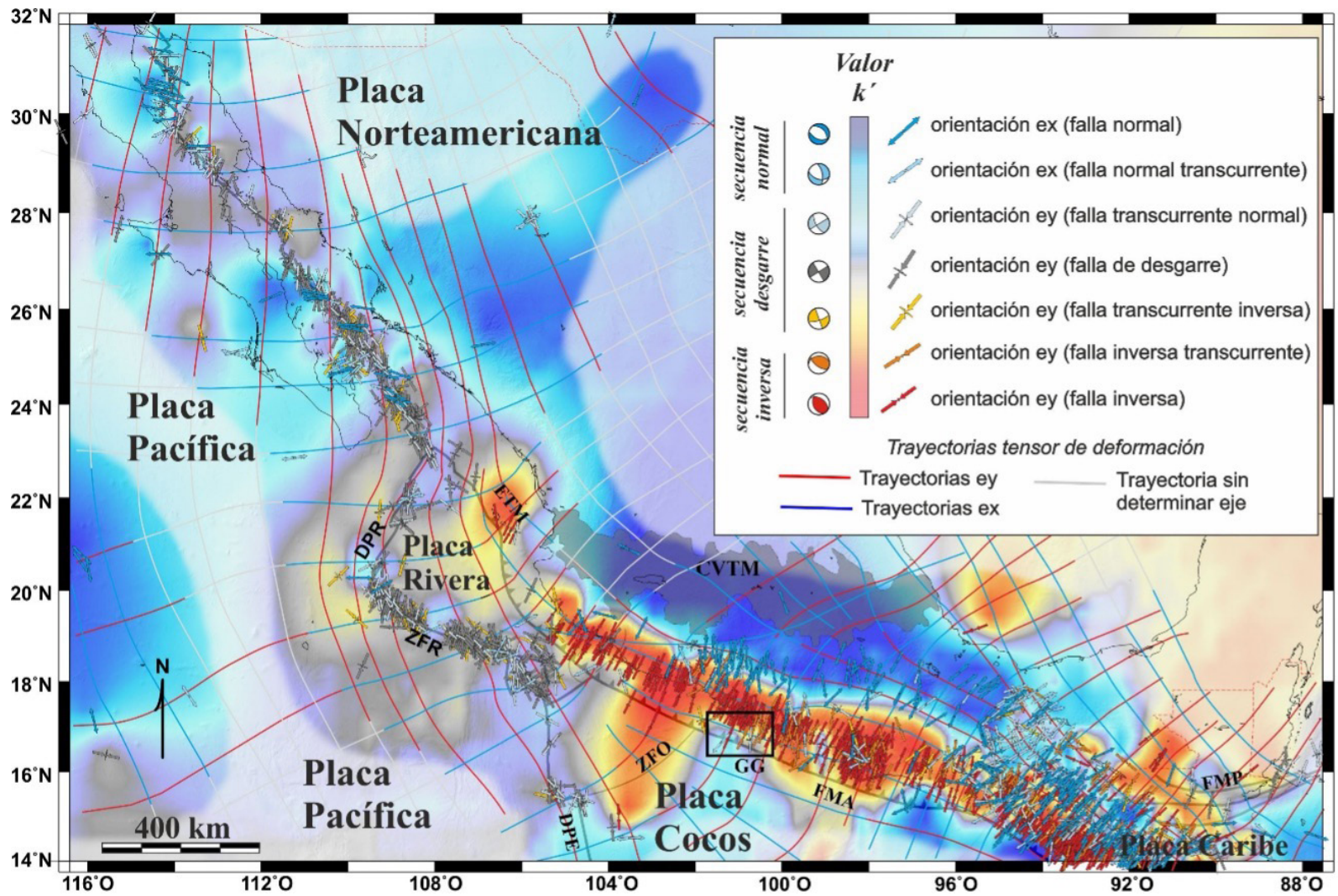

Figura 7 Resultados del análisis individual de los mecanismos focales mediante el MD (Capote et al., 1991; De Vicente, 1988). Se representan las direcciones de $e_{y}$ para la secuencia inversa y de desgarre, $y$ las orientaciones de $e_{x}$ para la secuencia normal. Se incluye el mapa interpolado de los valores puntuales de $k^{\prime}$ obtenidos del análisis de los mecanismos. Trayectorias de máximo ( $\left.e_{y}\right)$ y mínimo $\left(e_{x}\right)$ acortamiento horizontal construidas a partir de la interpolación de las orientaciones del tensor de deformación. GG Gap de Guerrero. 
Pacífico Este); zona 3b (zona NO de la fosa en el límite RIV-NAM); en la zona central de la fosa se localizan las zonas 3c y 4 a (caracterizadas por mecanismos normales), y las zonas $3 \mathrm{~d}$ y $4 \mathrm{~b}$ (mecanismos inversos); en la fosa en la zona más al SE del área de análisis se sitúan las zonas 4c y 5b (mecanismos inversos), 5a (mecanismos normales); y finalmente la zona 5c (en el límite CA-NAM).

La representación en el diagrama de Kaverina de estas poblaciones muestra los cambios en las tipologías de los mecanismos focales en las diferentes zonas (Figura 10). Las subpoblaciones situadas en el límite PA-RIV (2a, 2b, 2c, 2d) y en el límite PA-CG (3e) no muestran diferencias observables en la tipología de los mecanismos, y todas ellas están caracterizadas mayoritariamente por fallas de desgarre, y de desgarres normales e inversos. Los desgarres inversos están presentes en prácticamente todas estas subpoblaciones y llama la atención la ausencia casi total de mecanismos normales o normal transcurrentes. Es interesante destacar las diferencias entre estas poblaciones (desgarres y transcurrentes normales e inversos) y las definidas en la zona del Golfo de California (desgarres, transcurrentes normales y mecanismos normales transcurrentes y normales) (Figura 8). El resto de las subpoblaciones se sitúan en la FMA, donde es destacable el aumento del número de fallas normales y transcurrentes desde el NO hacia el SE de la FMA. También cabe destacar la complejidad que transmite el diagrama de la zona 5a, que no presenta tipologías predominantes.

Una vez realizada la zonificación se ha procedido a analizar las distintas subpoblaciones de mecanismos con los dos métodos de análisis de la deformación: MD y DR; obteniendo resultados para el conjunto de datos asignado a cada una de las zonas analizadas (Figura 11).

Para poder establecer las variaciones del tensor de deformación (cambios en la orientación, permutaciones de ejes) se ha procedido a la construcción de las trayectorias de deformación mediante el programa Lissage (Lee y Angelier, 1994). En la mayoría de los trabajos se suele representar únicamente la orientación puntual de $\mathrm{S}_{\mathrm{H} \max }$ o e e $\mathrm{e}_{\mathrm{y}}$ En este trabajo se representan las trayectorias de ey
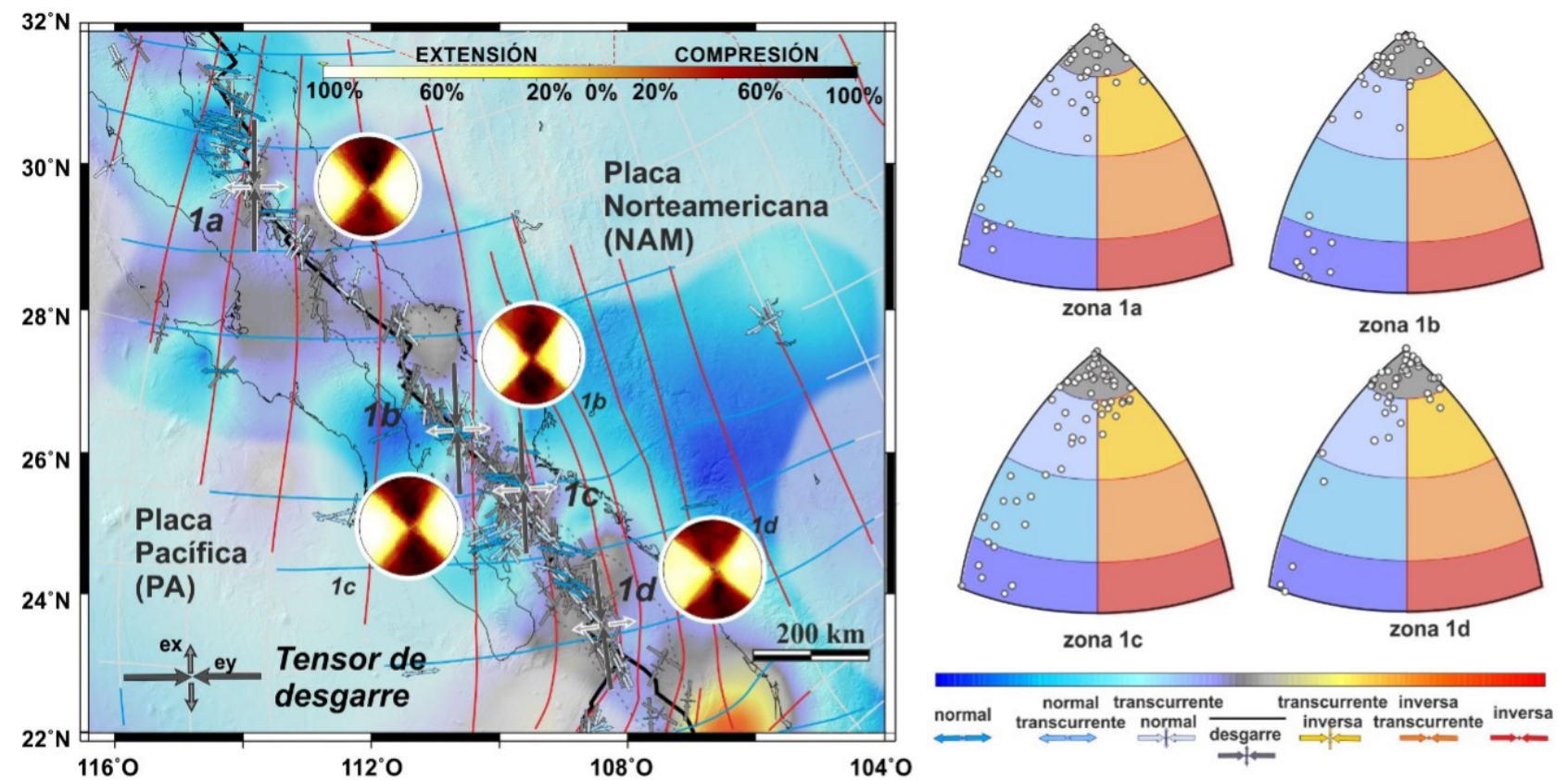

Figura 8 Análisis del límite de la Placa Pacífica con la Placa Norteamericana en el área más noroccidental de la zona de estudio (Golfo de California). Diagramas de Diedros Rectos y orientación del Tensor de Deformación (MD). Toda la zona muestra una deformación compatible con un tensor en desgarre donde $e_{y}$ se orienta N-S en las zonas 1a, 1b y 1c, con una suave rotación en la zona $1 \mathrm{~d}$. Los diagramas de Kaverina muestran un máximo de terremotos en desgarre con cierta componente extensional. Los diedros rectos por zonas son bastante homogéneos en desgarre con simetría ortorrómbica. 
y e $\mathrm{e}_{\mathrm{x}}$ (Figura 12), es decir la orientación de los ejes principales de la elipse resultante de la proyección del elipsoide de deformación en la horizontal. Este tipo de representación permite discriminar claramente las variaciones en la orientación del tensor, relacionadas con las grandes estructuras y límites, de las posibles permutaciones de ejes que pueden estar relacionadas con diferentes efectos locales o regionales.

El análisis del factor $\mathrm{k}^{\prime}$ se ha representado bidimensionalmente en el mapa. No obstante, podemos establecer también un análisis tridimensional de la variación del factor k'. Para ello, se han realizado 14 perfiles seriados en el límite de la NAM con las placas de RIV, CG y CA (Figura 13) en los que se muestra la distribución de la deformación en profundidad.

\section{Discusión}

Los resultados obtenidos sobre el estado de deformación por zonas en nuestro análisis son similares a los obtenidos por Suter (1991). No obstante, en algunas zonas, como en la provincia b definida por este autor (Figura 2), se identifican algunas diferencias en la orientación del tensor que en ambos casos es extensivo. Los resultados de orientación del tensor (trayectorias de $\mathrm{e}_{\mathrm{v}}$ ) (Figura 7) muestran una orientación muy homogénea de los dos ejes

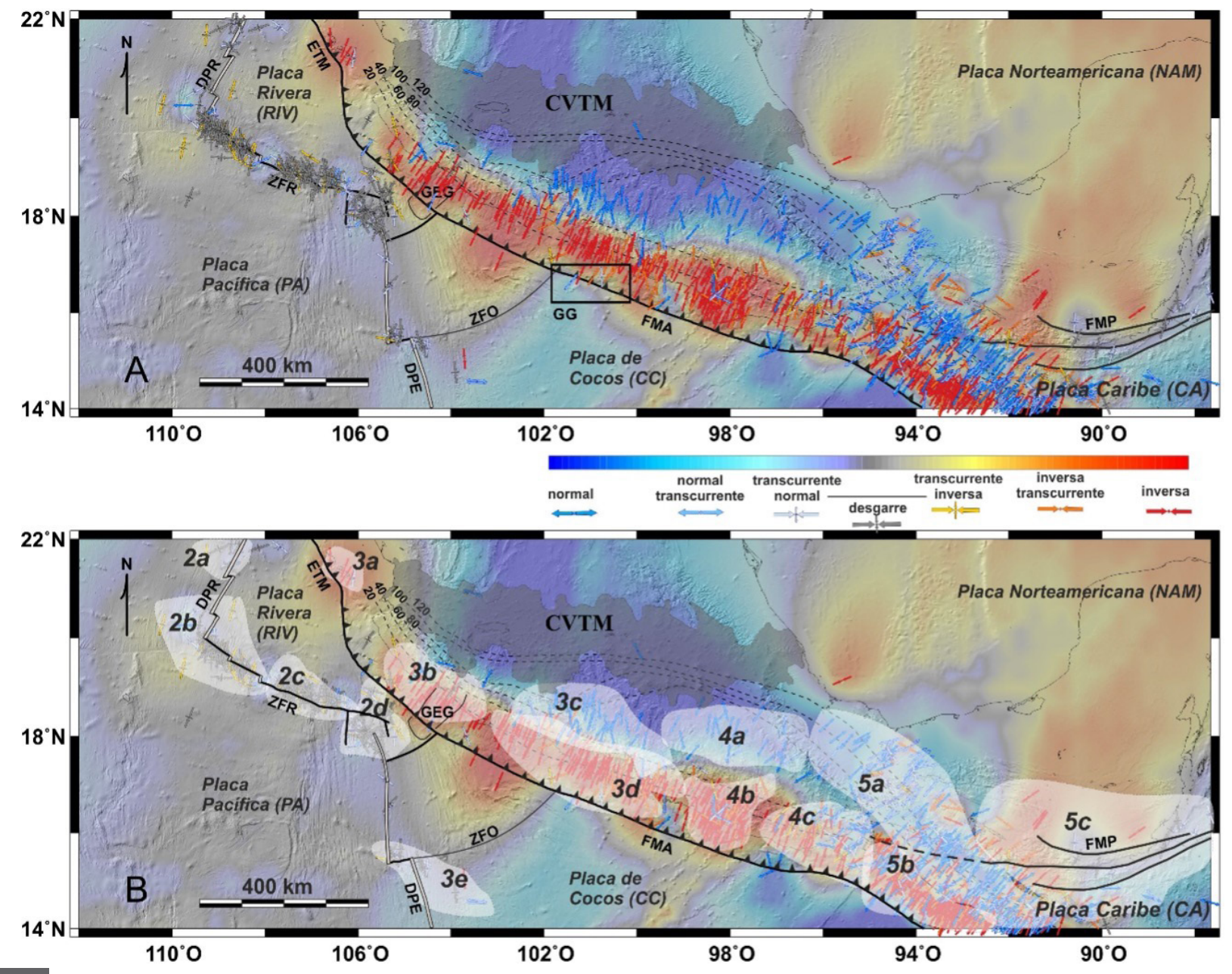

Figura 9 A) Distribución individual de orientaciones de e deducidas del análisis de la Fosa Mesoamericana. Mapa de factor de forma del tensor de deformación ( $\left.k^{\prime}\right)$. GG Gap de Guerrero. B) Definición de zonas en función de la distribución espacial y la tipología de los mecanismos focales. Geometría de la lámina de subducción según Pardo y Suárez (1995). 
del tensor (según NE-SO y NO-SE), mientras que los propuestos por Suter (1991) muestran en algunas zonas los ejes que aparecen orientados según N-S y E-O (extensión E-O).

En cuanto al World Stress Map (WSM16; Heidbach et al., 2016a, 2016b; último acceso el 1 de septiembre, 2020), éste aporta datos adicionales a los mecanismos, siendo en esta zona fundamentalmente datos obtenidos en sondeos o de la interpretación de datos geológicos (alineación de focos volcánicos, etc). Estos datos son muy útiles para completar zonas en las que no tenemos datos de mecanismos focales que nos permitan analizar adecuadamente alguna de las zonas. En este caso,
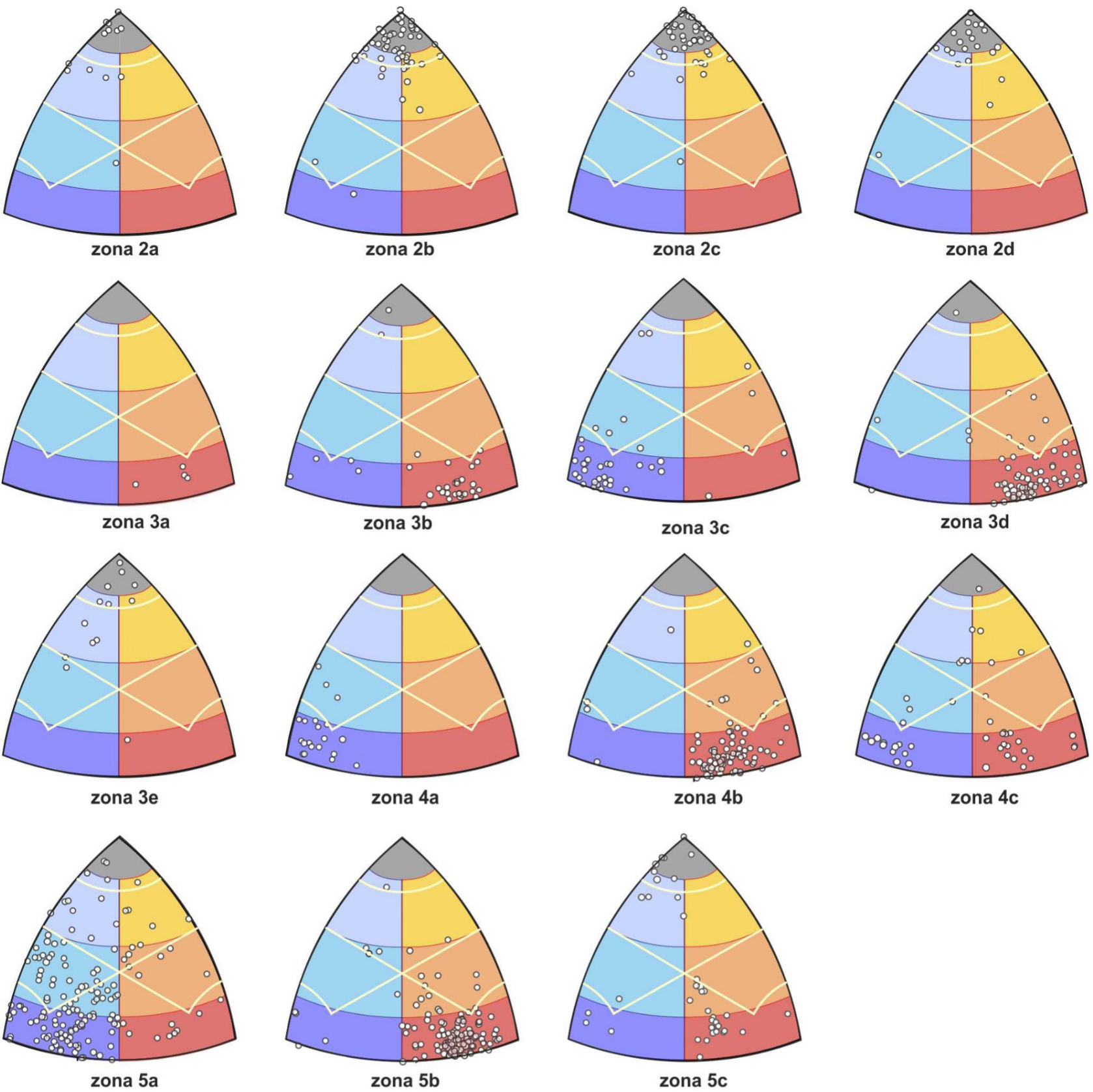

Figura 10 Diagramas ternarios (Kaverina) de las zonas establecidas en la zona de la Fosa Mesoamericana. Las zonas corresponden a las definidas en la figura 9B. La separación por zonas corresponde a los diferentes grupos de datos agrupados espacialmente a partir del análisis cluster (ver leyenda en Figura 6). 
la correlación entre los datos del WSM (Figura 2) y los resultados de nuestro análisis (Figura 7) (interpolación del factor de k' y orientación de las trayectorias de deformación), muestra una buena correlación. No obstante, en algunas zonas, como en el CVTM, surgen algunas discrepancias, ya que los datos del WSM muestran una orientación de $\mathrm{S}_{\mathrm{Hmax}}$ según E-O, mientras que nuestro análisis muestra una orientación que varía de ENE-OSO a E-O, si bien es cierto que el tipo de datos del WSM en esta zona son casi exclusivamente alineaciones de focos volcánicos, sujetos siempre a posibles interpretaciones.

El tipo de análisis implementado en este trabajo permite completar otros tipos de análisis, ya que la información que proporciona la interpolación de la forma (mapa de k') y orientación del tensor de deformación (trayectorias) permite analizar con más detalle las variaciones de estos parámetros tanto en 2 como en 3 dimensiones.

Los diagramas ternarios de Kaverina permiten estudiar el estilo de la deformación en cada límite de placa (Figura 6): en la zona NO (Figura 6a), el límite de placa PA-NAM presenta un estilo en desagarre con componente normal, en consonancia con los grandes desgarres del Golfo de California, mientras que los límites PA-RIV (Figura 6b), muestran un estilo en desgarre con componentes normales e inversas por igual; en ambos límites la densidad de terremotos por km2
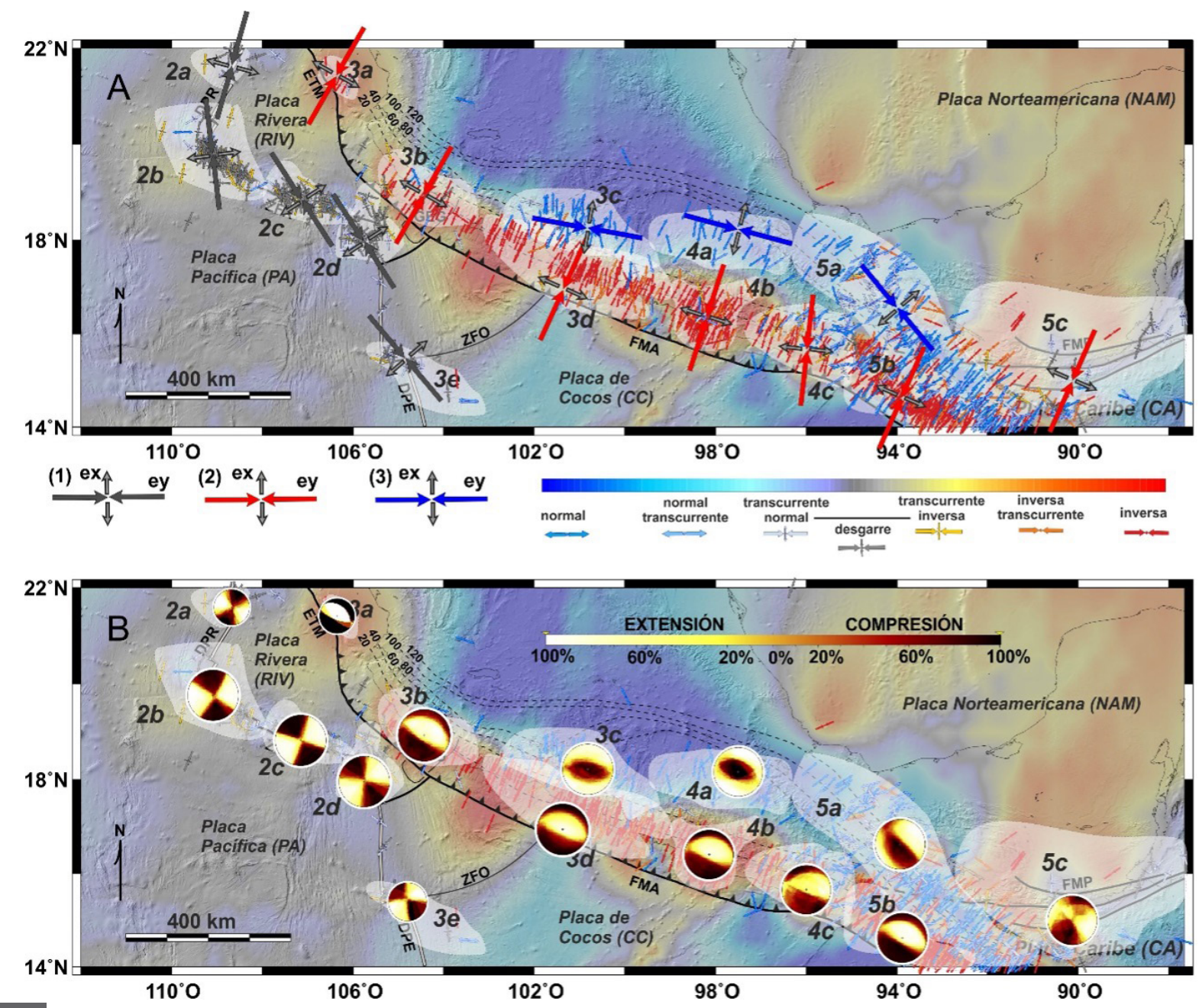

Figura 11 Análisis de la deformación por zonas utilizando: A) Modelo de Deslizamiento (MD) donde se representa la orientación de la proyección de la elipse del tensor de deformación en la horizontal $\left(e_{y}\right.$ y $\left.e_{x}\right)$. B) Diedros Rectos (DR), donde se representan los diagramas de diedros rectos según el análisis cluster y agrupados por las zonas definidas en la figura 9B. Geometría de la lámina de subducción según Pardo y Suárez (1995). (1) Tensor de desgarre, (2) tensor compresivo, (3) tensor extensivo. 
es elevada. En cuanto al límite PA-CG, la densidad de terremotos con mecanismo focal es baja, con un estilo en desgarre con componente extensional (Figura 6c). Sin embargo, el límite RIV-NAM (Figura 6d) presenta una baja densidad de sismos, mayoritariamente inversos y relacionados con la zona NO de la FMA. La sismicidad instrumental muestra que la densidad de terremotos de la placa RIV es mayor en su límite con la placa PA frente a la placa NAM (Figuras 1, 5). El hecho de que además en el límite PA-RIV aparezcan terremotos inversos de subducción, cabría preguntarse si se está activando una segunda lámina de subducción en la zona, concretamente en la zona con orientación NO-SE. En cuanto al límite CG-CA (Figuras 6e, f, g), se observa una variación en los diagramas ternarios desde la zona NE hacia la zona SE, donde se encuentra con la Zona de Falla de Motagua-Polochic. La densidad de sismos aumenta desde el NO hacia el SE. Sin embargo, se observa también una transición hacia el SE desde el NO de la deformación compresiva, para valores más extensionales, lo que indica una mayor acomodación de la convergencia en la zona de Guerrero (zona centro) frente a la zona de Oaxaca (zona SE) y confluencia con la placa CA (Figura $6)$.

\subsection{ANÁLISIS "GLUSTER" 2D}

A partir del análisis cluster de los mecanismos focales, la distribución espacial de las zonas de deformación y del factor de forma (k') (Figura 7), se pueden estimar los límites de ruptura 2D en la FMA. Por sectores, en el Golfo de California dominan los terremotos en desgarre con componente normal (Figura 6a) y no parecen observarse diferencias importantes ni en la orientación de $e_{\mathrm{v}}$, ni en la tipología predominante de mecanismos focales. En la placa RIV, solo aparecen terremotos con mecanismo focal inverso en el Escarpe Tres Marías (ETM, Figura 7), mientras que, al sur, en la zona de subducción RIV-NAM, apenas hay terremotos de magnitud superior a M 5. Obviamente, el giro de la subducción frente al ETM provoca un cambio en el tensor de deformación que, si bien

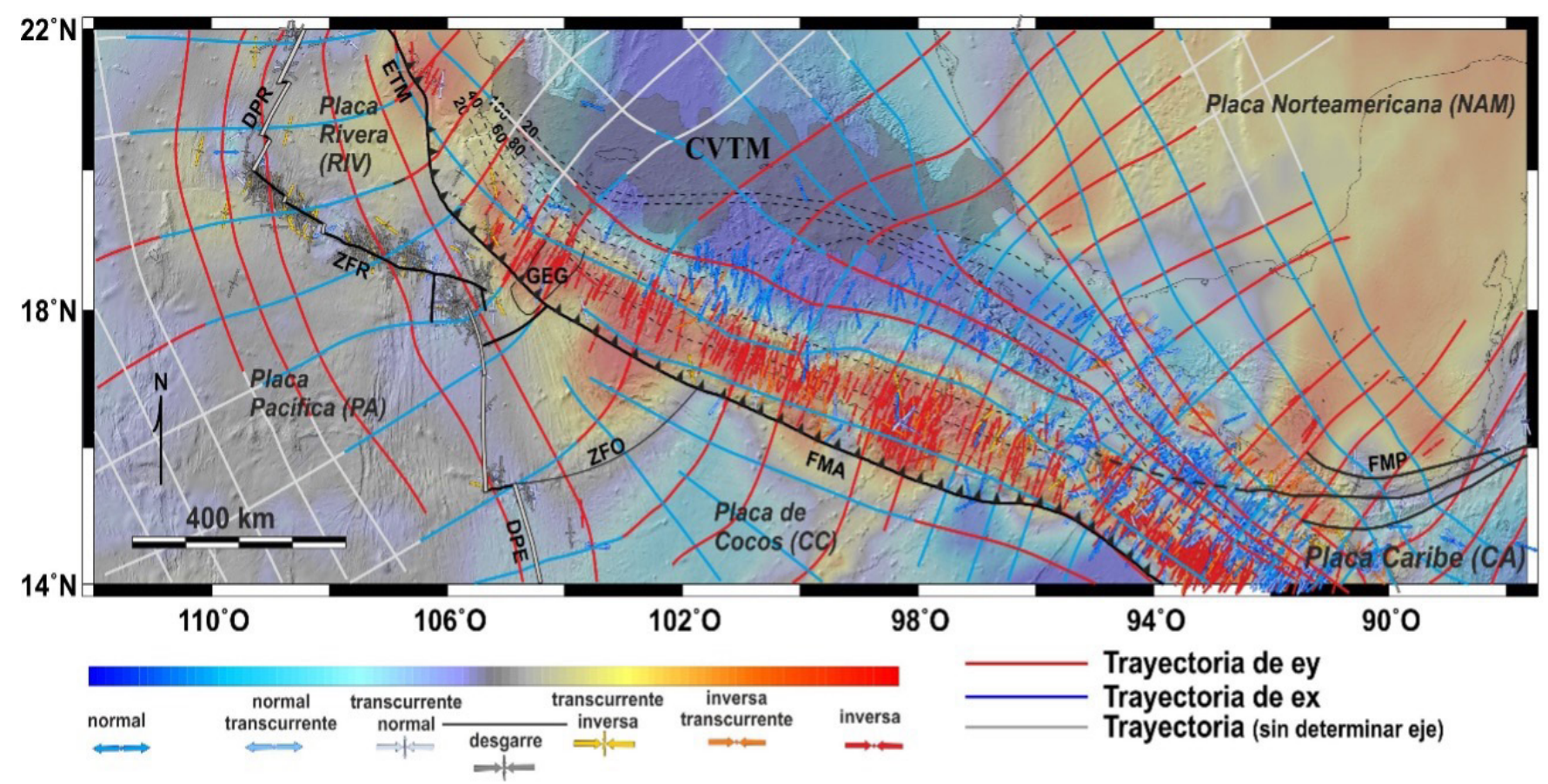

Figura 12 Representación de las trayectorias de deformación interpoladas a partir de los datos individuales de e deducidos del análisis mediante el Modelo de Deslizamiento, en relación a los principales límites de placa y la geometría de la lámina de subducción, a partir del modelo presentado por Pardo y Suárez (1995). 

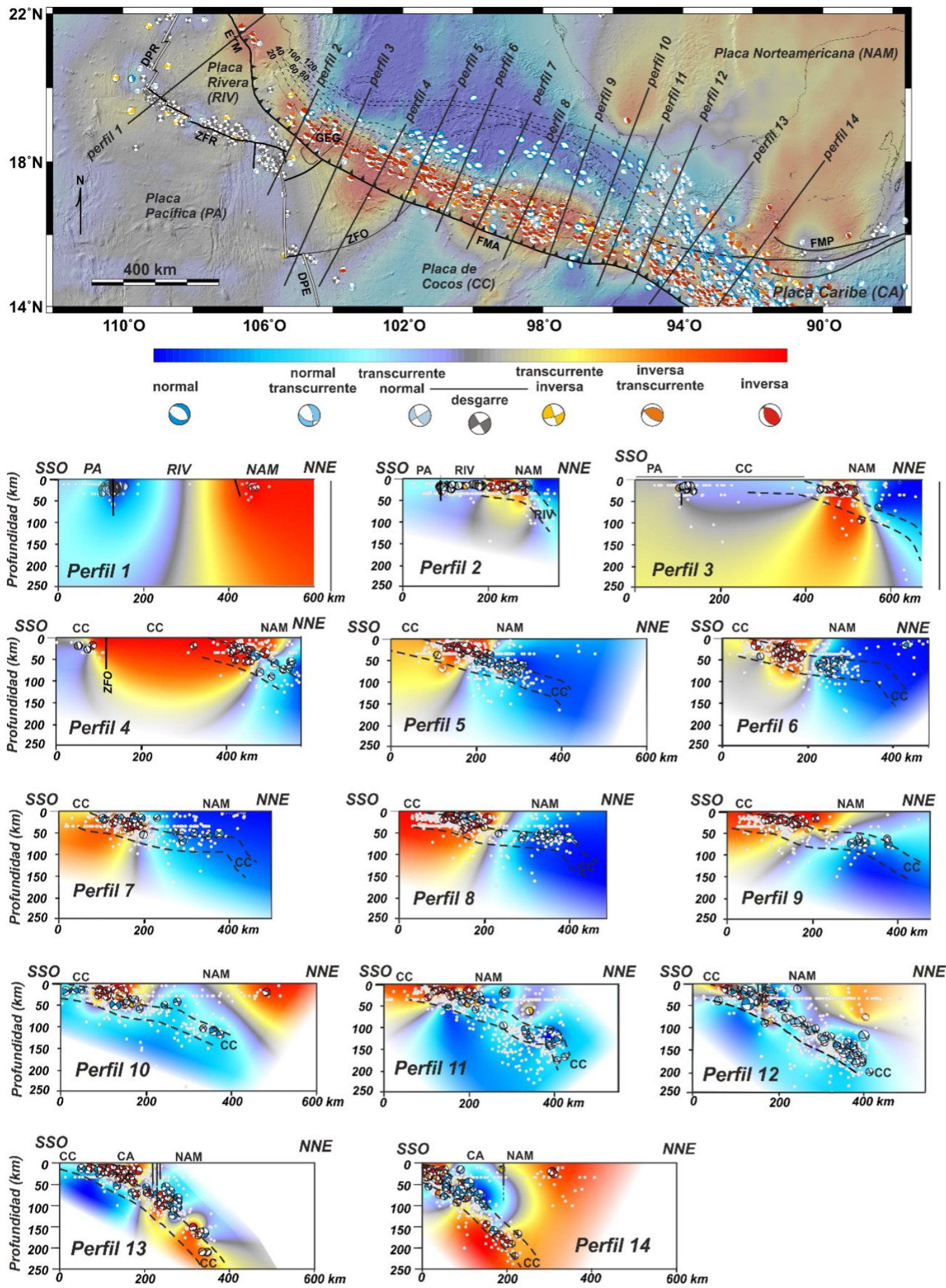

Figura 13 Cortes seriados en la Fosa Mesoamericana con la distribución de los mecanismos focales (la tipología de los mecanismos viene definida por su color, ver leyenda en Figura 5 ) y mapa del factor de forma del tensor de deformación ( $\left.k^{\prime}\right)$ y su interpolación en profundidad. Se ha representado la geometría de la lámina de subducción con una línea discontinua a partir del modelo de Pardo y Suárez (1995). Las zonas blancas representan zonas sin datos de interpolación. 
no es un cambio en la forma del tensor, sí lo sería en su orientación. Esto podría explicar la escasa incidencia de sismos en la zona, o bien que sus periodos de retorno sean mayores que en la zona de ETM. También el hecho de que parte de la convergencia se acomode entre las placas PA-RIV, reduciría la cantidad de deformación compresiva que estaría absorbiendo el límite RIV-NAM, tal y como se ha discutido antes.

En cuanto al análisis 3D de la deformación en la FMA en la placa de CC, a pesar de que la subducción tiene la misma orientación, observamos una clara influencia de la Zona de Falla de Orozco (ZFO) que compartimenta la deformación 2D.

En detalle, el análisis del límite de PA-NAM en el área más noroccidental de la zona de estudio (Golfo de California, Figura 8), muestra una deformación compatible con un tensor en desgarre donde $\mathrm{e}_{\mathrm{y}}$ se orienta N-S en las zonas 1a, lb y lc, con una suave rotación en la zona $1 \mathrm{~d}$. Los diagramas de Kaverina muestran un máximo de terremotos en desgarre con cierta componente extensional. Los diedros rectos por zonas son bastante homogéneos con una simetría ortorrómbica.

En cuanto al análisis cluster 2D de la FMA (Figura 9), los tensores de deformación aparecen fuertemente condicionados por la geometría de la lámina de subducción, según el modelo de Pardo y Suárez (1995). Se han agrupado por zonas homogéneas a partir de la interpolación 2D del k', destacando la existencia de zonas diferentes en la FMA, concretamente las zonas 3d, 4b y 4c (Figura 9). Los diagramas ternarios de Kaverina permiten caracterizar la transición 2D de los tensores de deformación en relación a los límites de placa y a la distribución espacial de la deformación (Figura 10). A partir de las zonas definidas por el análisis cluster, se observa una transición desde el NO al SE en la FMA. Por un lado, se observa una transición con respecto a la tipología de los terremotos: de terremotos predominantemente compresivos en el $\mathrm{NO}$ (zona 3d) a zonas con un aumento progresivo de terremotos de tipo extensivo y de desgarre (4c) (Figuras 6 y 11). Por otro lado, también se puede definir una rotación progresiva de la orientación de $e_{y}$ en sentido anti-horario (Figura 11) desde la zona NO con $\mathrm{e}_{\mathrm{y}}$ según NE-SO (zona 3d), hacia el SE de la FMA, con e según NNE-SSO (zona 4c). Este tipo de variaciones en la orientación de $\mathrm{e}_{\mathrm{y}} \mathrm{y}$ en la tipología de los mecanismos nos está mostrando el cambio en el buzamiento de la lámina de subducción que se produce en la zona 4c.

\subsection{ANÁLISIS 3D DE LA DEFORMACIÓN}

El estudio de la deformación sísmica a partir del análisis cluster de la deformación (DR y MD), también permite realizar un análisis tridimensional de la distribución de la deformación. Para ello, se han llevado a cabo una serie de perfiles del valor de k' que muestran las variaciones de la deformación en profundidad (Figura 13). En total, se han realizado 14 perfiles donde se muestra la distribución en profundidad de los mecanismos focales y se interpola el factor de forma del tensor de deformación (k'). La robustez de este análisis permite analizar la transición en profundidad de la deformación sísmica, lo que permite analizar la existencia de zonas acopladas y desacopladas, la presencia de corrientes mantélicas y las transiciones de las zonas de convergencia con las zonas extensionales en la lámina de subducción. En el perfil 1 (Figura 13) no se observa un proceso de subducción bien definido, el límite RIV-NAM está caracterizado únicamente por fallas de carácter inverso en el Escarpe Tres Marías (ETM). Los perfiles 2 y 3, empiezan a mostrar un incipiente cambio de buzamiento de la lámina, pero no se observa un desacoplamiento evidente en profundidad. En los perfiles del 4 al 9 se puede establecer la presencia de un desacoplamiento situado entre los 50 y los $75 \mathrm{~km}$ de profundidad, caracterizado por sismos de carácter normal y normal transcurrente. Este desacoplamiento parece coincidir con la marcada horizontalización de la lámina (perfiles del 6 al 9). En los perfiles 10 y 11, la zona de desacople (caracterizada también por sismos de la secuencia normal) parece situarse algo más profunda (alrededor de los $100 \mathrm{~km}$ de profundidad), a la vez que la lámina parece aumentar su buzamiento. El perfil 12 parece definir una zona de transición a la zona de los perfiles 13 y 14, en los que se observa 
un acoplamiento en profundidad de la Placa de Cocos caracterizado por la existencia de una zona de compresión profunda (aproximadamente a los $150 \mathrm{~km}$ de profundidad), posiblemente relacionado con una corriente mantélica (Castellanos et al., 2018; Carciumaru et al., 2020).

También podemos destacar que, la zona de división del tensor de deformación a partir del k' (Figura 7), coincidiría con la denominada como Laguna Sísmica ( $g a p$ ) de Guerrero de la FMA de la convergencia CG-NAM (Kostoglodov et al., 1996), lo que podría determinar la ocurrencia de los denominados terremotos silenciosos (Kostoglodov et al., 1996, 2003). Por otro lado, la distribución del tensor de deformación a lo largo de la lámina de subducción CG-NAM (Figura 14) coincide con la distribución de las propiedades friccionales propuestas por Radiguet et al. (2016), como mecanismo de ocurrencia de terremotos lentos en la zona de la laguna sísmica de Guerrero.

La zona del gap de Guerrero (GG), en la FMA, aparece delimitada por el factor de forma del tensor de deformación, k', tanto en la distribución bidimensional como en los cortes en profundidad.
La Figura 7 muestra como el GG queda delimitado por izquierda y derecha por un k' en desgarre más próximo al límite de la trinchera de la FMA, en la proyección sobre este límite donde se produce la permutación de e que cambia de NNE a ONO en profundidad, dominado por terremotos en fallamiento normal entre 60 y $100 \mathrm{~km}$ de profundidad.

En cuanto a la distribución en profundidad, el GG se localiza entre los perfiles 6, 7, 8 y 9, justo donde se produce un cambio de la lámina de subducción, separando la zona de transición de convergencia a extensión a partir de $50 \mathrm{~km}$ de profundidad, apareciendo la lámina de subducción plana (flat slab).

Según los resultados obtenidos, aparece: a) una convergencia geométrica entre la variación 3D del $\left.\mathrm{k}^{\prime}, \mathrm{b}\right)$ un cambio de zona compresiva a extensional a partir de $50 \mathrm{~km}, \mathrm{c}$ ) un cambio de las propiedades friccionales de la lámina de subducción (Radiguet et al., 2016) y d) la existencia de una zona donde podrían concentrarse terremotos lentos y que podría ejercer de barrera para romper por completo el segmento Michoacán-Oaxaca de la FMA en un único evento.
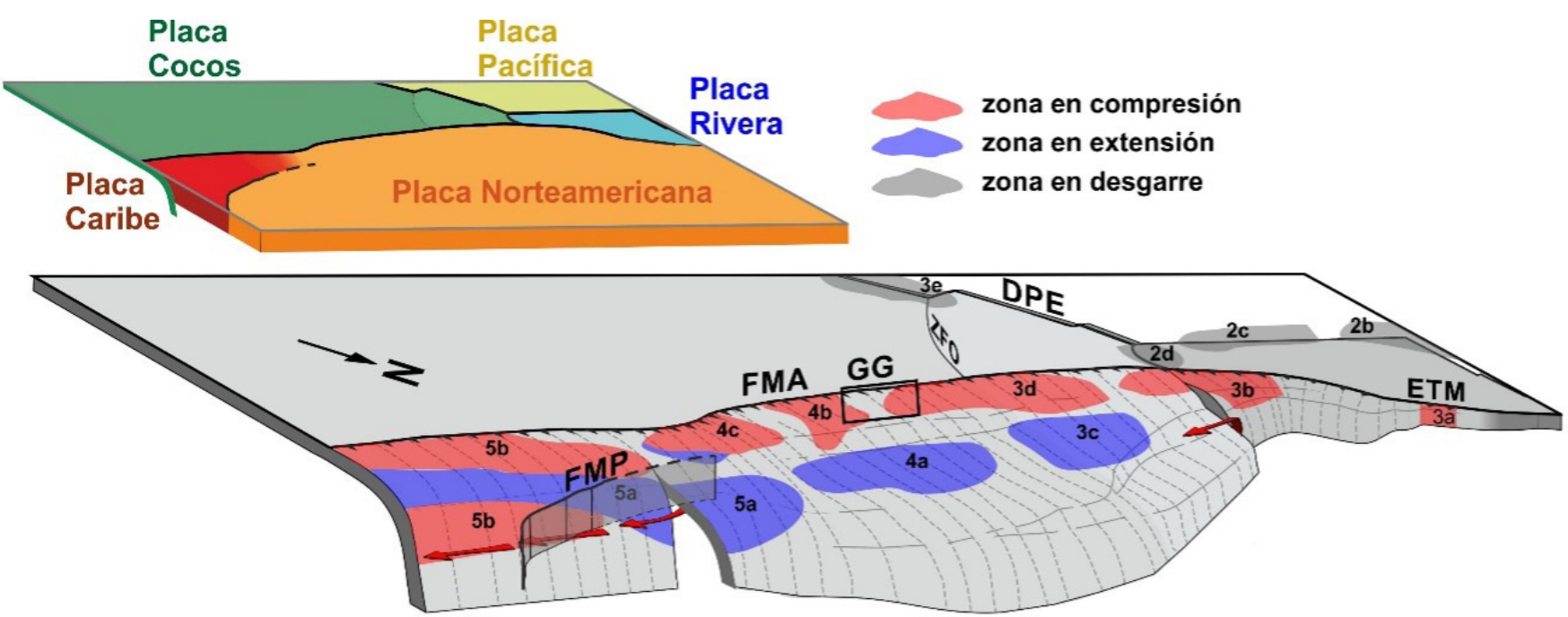

Figura 14 Modelo geométrico conceptual de subducción compleja para las placas de Cocos y Rivera bajo la Placa Norteamericana. Se representan por zonas 3D los estilos de deformación obtenidos del análisis cluster (zonas extensionales, compresivas y en desgarre), así como otras propiedades como la posición y la dirección de movimiento del flujo mantélico (flechas rojas, Yang et al., 2009; Castellanos et al., 2018; Carciumaru et al., 2020). GG Gap de Guerrero. 


\section{Conclusiones}

El análisis cluster 3D de los tensores de deformación obtenido mediante el análisis de los Diedros Rectos y Modelo de deslizamiento de los mecanismos focales permite cartografiar la deformación en zonas sísmicamente activas. La aplicación en la Fosa Mesoamericana (FMA) en la zona de influencia mexicana muestra:

1. El Golfo de California acomoda principalmente la deformación tectónica mediante terremotos en desgarre con componente extensional.

2. El límite convergente entre la Placa de Rivera con la Placa Pacífica podría explicar la baja incidencia de grandes terremotos inversos en el límite de la Placa Rivera con la Placa Norteamericana.

3. El límite convergente de la Placa de Cocos con la Placa Norteamericana presenta diferentes estilos de la deformación asociados a la variación de la geometría de la FMA. Esta variación de la deformación obedece al cambio de buzamiento de la subducción, en forma de rampa - rellano sub-horizontal. A partir de los 60-80 km de profundidad, aparece un desacoplamiento de la subducción mostrando solo terremotos de tipo normal en los perfiles, lo que sugiere que la velocidad de convergencia CG-CA es inferior a la velocidad de arrastre (pulling) de la parte terminal de la subducción por debajo de $100 \mathrm{~km}$ de profundidad.

4. La zona de transición entre los segmentos de Michoacán y Oaxaca podría explicar la laguna sísmica (gap) de Guerrero como una zona inter-segmento que podría favorecer la ocurrencia de los denominados terremotos lentos y silenciosos.

5. La existencia de corrientes mantélicas que zigzaguean entre la lámina de subducción aprovechando los cambios de buzamiento y estilo de la deformación, genera ventanas litosféricas a partir de $100 \mathrm{~km}$ de profundidad y podría jugar un papel importante tanto en el desacoplamiento de la subducción a partir de esa profundidad, como en el estilo del vulcanismo tras-arco.

Finalmente, destacar que este tipo de estudios de la deformación 3D a partir de los tensores de deformación, puede ser muy útil para complementar los estudios presentes y futuros mediante técnicas de GPS, análisis de la anisotropía de la velocidad de la onda $\mathrm{P}$ y tomografía sísmica. La aportación de la metodología presentada en este trabajo y calibrada en otras zonas, puede ayudar a comprender mejor el ciclo sísmico para fuentes sismogénicas con capacidad de generar terremotos de M 8, así como la existencia de terremotos lentos en zonas inter-segmento.

\section{Agradecimientos}

Queremos agradecer la revisión realizada por dos autores anónimos que han mejorado la versión final de este manuscrito. Este trabajo ha sido financiado parcialmente por los proyectos del Plan Nacional de $\mathrm{I}+\mathrm{D}+\mathrm{i}$ del Ministerio de Ciencia e Innovación de España con referencias 3GEO CGL2017-83931-C3-2-P y MINECO-FEDER CGL2015-67169-P (QTECSPAIN). Este trabajo es una contribución del Grupo de trabajo QTECT-AEQUA.

\section{Referencias}

Álvarez, R., Yutsis, V., 2015, The elusive RiveraCocos plate boundary: not diffuse. En Wright, T. J., Ayele, A., Ferguson, D. J., Kidane, T. y Vye-Brown, C. (eds), Magmatic Rifting and Active Volcanism: Geological Society, London, Special Publications, 420, http:// doi.org/10.1144/SP420.8

Álvarez-Gómez, J.A., 2014, FMG a one-liner Python program to manage, classify and plot focal mechanisms: Geophysical Research Abstracts, 16.

Álvarez-Gómez, J.A., 2019, FMC - Earthquake focal mechanisms data management, cluster and classification: SoftwareX, 9, 299-307. https://doi.org/10.1016/j. softx.2019.03.008

Álvarez-Gómez, J.A., Staller Vázquez, A., Martínez-Díaz, J.J., Canora, C., 
Alonso-Henar, J., Insua-Arévalo, J.M., BéjarPizarro, M., 2019, Push-pull driving of the Central America Forearc in the context of the Cocos-Caribbean-North America triple junction: Scientific Reports 9, 11164 . https:// doi.org/10.1038/s41598-019-47617-3

Angelier, J., Mechler, P., 1977, Sur une méthode graphique de recherche des contraintes principales également utilisables en tectonique et en sismologie: la méthode des diedres droits: Bulletin de la Société Géologique de France, S7-19 (6), 13091318. https://doi.org/10.2113/gssgfbull. S7-XIX.6.1309

Capote, R., DeVicente, G., González-Casado,J.M., 1991,An application of theslip model of brittle deformations to focal mechanism analysis in three different plate tectonics situations: Tectonophysics, 191, 339-409. https://doi. org/10.1016/0040- 1951(91)90070-9

Carciumaru, D., Ortega, R., Castellanos, J. C., Huesca-Pérez, E., 2020, Crustal characteristics in the subduction zone of Mexico: implication of the tectonostratigraphic terranes on slab tearing: Seismological Research Letters, 91(3), 17811793. https://doi.org/10.1785/0220190117 Castellanos, J.C., Clayton, R.W., Pérez-Campos, X., 2018, Imaging the Eastern TransMexican Volcanic Belt WithAmbient Seismic Noise: Evidence for a Slab Tear: Journal of Geophysical Research: Solid Earth, 123 (9), 7741-7759. https://doi. org/10.1029/2018JB015783

CMT, Global Centroid-Moment-Tensor, 2020, The Global CMT Project: National Science Foundation,Virginia, EEUU, actualizado 30 de junio de 2020, disponible en https:// www.globalcmt.org/

Corbo-Camargo, F., Arzate-Flores, J.A., AlvarezBéjar, R., Aranda-Gómez, J.J., Yutsis V., 2013, Subduction of the Rivera plate beneath the Jalisco block as imaged by a magnetotelluric data: Revista Mexicana de Ciencias Geológicas, 30(2), 268-281.
DeMets, G., Wilson, D., 1997, Relative motions of the Pacific, Rivera, North American, and Cocos plates since 0.78 Ma: Journal of Geophysical Research, 102, 2789-2806. https://doi.org/10.1029/96jb03170

DeMets, C., Gordon, R.G., Argus, D.F., Stein, S., 1990, Current plate motions, Geophysical Journal International, 101, 425-478. https://doi.org/10.1111/j.1365-246x. 1990. tb06579.x

DeMets, C., Gordon, R. G., Argus D. F., 2010, Geologically current plate motions: Geophysical Journal International, 181(1), 1-80. https://doi. org/10.1111/j.1365-246X.2009.04491.x

De Vicente, G., 1987, The e/k' diagram. An application of the slip model to the population fault analysis: Revista de la Sociedad Geológica de España, 1, 97-112.

De Vicente, G., 1988, Análisis poblacional de Fallas. El sector de enlace Sistema CientralCordillera Ibérica, Universidad Complutense de Madrid,Tesis Doctoral, $150 \mathrm{p}$.

De Vicente, G., Muñoz Martín, A., Giner, J., 1992, El uso del método de los diedros rectos. Implicaciones a partir del modelo de deslizamiento del análisis poblacional de fallas: Revista de la Sociedad Geológica de España, 5 (3-4), 7-19.

De Vicente, G., Cloetingh, S., Muñoz-Martín, A., Olaiz, A., Stich, D., Vegas, R., Galindo-Zaldívar, J., Fernández-Lozano, J., 2008, Inversion of moment tensor focal mechanisms for active stresses around the microcontinent Iberia: Tectonic implications: Tectonics, 27, TC1009, https://doi:10.1029/2006TC002093.

Duputel, Z., Rivera, L., Fukahata, Y., Kanamori, H., 2012, Uncertainty estimations for seismic source inversions: Geophysical Journal International, 190, 1243-1256, https://doi. org/10.1111/j.1365-246X.2012.05554.x

Ekström, G., Nettles, M., Dziewonski, A.M., 2012, The global CMT project 20042010: Centroid-moment tensors for 13,017 
earthquakes: Physics of the Earth and Planetary Interiors, 200-201, 1-9, http:// dx.doi.org/10.1016/j.pepi.2012.04.002

Fasola, S., Brudzinski, M. R., Ghouse, N., Solada, K., Sit, S., Cabral-Cano, E., ArciniegaCeballos, A., Kelly, N., Jensen, K., 2016, New perspective on the transition from flat to steeper subduction in Oaxaca, Mexico, based on seismicity, nonvolcanic tremor, and slow slip: Journal of Geophysical Research, 121, 1835-1848. https://doi. org/10.1002/2015JB012709

Ferrari, L., 2004, Slab detachment control on mafic volcanic pulse and mantle heterogeneity in central Mexico: Geology, 32, 77-80. https:// doi.org/10.1130/G19887.1

Ferrari L., Orozco-Esquivel T., Manea, V., Manea, M., 2012, The dynamic history of the TransMexican Volcanic Belt and the Mexico subduction zone: Tectonophysics, 522523(5), 122-149. https://doi.org/10.1016/j. tecto.2011.09.018

Frohlich, C., Apperson, K.D., 1992, Earthquake focal mechanisms, moment tensors, and the consistency of seismic activity near plate boundaries: Tectonics, 11, (2), 279-296. https://doi.org/10.1029/91TC02888

Gardi, A., M. Cocco, A. M. Negredo, R. Sabadini, S. K. Singh., 2000, Dynamic modelling of the subduction zone of central Mexico: Geophysical Journal International 143(3), 809-820. https://doi. org/10.1046/j.1365-246X.2000.00291.x

Giner-Robles, J.L., González-Casado, J.M., Gumiel, P., Martín-Velázquez, S., GarcíaCuevas, C., 2003a, A kinematic model of the Scotia plate (SW Atlantic Ocean): Journal of South American Earth Sciences, 16 (4), 179-191. https://doi.org/10.1016/ S0895-981 1(03)00064-6

Giner-Robles, J.L., González-Casado, J.M., Gumiel, P., Martín-Velázquez, S., GarcíaCuevas, C., 2003b, Changes in strain trajectories in three different types of plate tectonic boundary deduced from earthquake focal mechanisms: Tectonophysics, 372, 179-191. https://doi.org/10.1016/ S0040-1951(03)00310-X

Giner-Robles, J.L, Gumiel, P., Pérez-López, R., Rodríguez-Pascua, M.A., GarcíaMayordomo, J., Paredes, C., GonzálezCasado, J.M., 2006, Relevance of the fault plane correct orientation regarding the focal mechanism analysis of earthquakes (resumen), en XIII Asamblea Nacional y $5^{\text {a }}$ Hispano-Portuguesa de Geodesia y Geofísica: Sevilla (España), Congress Proceedings, Sevilla, España, Instituto Geográfico Nacional, 1-4.

Giner-Robles, J.L., Pérez-López, R., MartínezDíaz, J.J., Rodríguez-Pascua, M.A., González-Casado, J.M., 2008, Cluster analysis of the 3D instrumental seismicity within a subduction zone (Caribbean, Cocos and North American tectonic convergence). Implications for a kinematical tectonic model, in $33^{\text {rd }}$ International Geological Congress: Oslo, Noruega, International Union of Geological Sciences.

Giner-Robles, J.L., Pérez-López, R., RodríguezPascua, M.A., Martínez-Díaz, J.J., GonzálezCasado, J.M., 2009, Present-day strain field on the South American slab underneath the Sandwich Plate (Southern Atlantic Ocean): a kinematic model: Geological Society London, 328(1), 155-167. https://doi.org/10.1144/ SP328.6

Gómez-Tuena, A., Orozco-Esquivel, M. T., Ferrari, L., 2007, Igneous petrogenesis of the Trans-Mexican Volcanic Belt. In: Alaniz-Álvarez, S.A., and NietoSamaniego, Á.F., eds. Geology of México: Celebrating the Centenary of the Geological Society of México: Geological Society of America, 422, 129-181. https://doi. org/10.1130/2007.2422(05)

Gómez-Tuena, A.G., Mori, L., Straub, S.M., 2018, Geochemical and petrological insights into the tectonic origin of the Transmexican Volcanic Belt: Earth-Science Reviews, 
183, 153-181. https://doi.org/10.1016/j. earscirev.2016.12.006

González-Casado,J.M., Giner-Robles,J.L., LópezMartínez,J., 2000, Bransfield Basin, Antarctic Peninsula: Not a normal backarc basin: Geology, 28 (11), 1043-1046. https://doi. org/10.1130/0091-7613(2000)28<1043:BB $\mathrm{APNA}>2.0 . \mathrm{CO} ; 2$

Guzmán-Speziale, M., Gómez-González, J. M., 2006, Seismic Strain rate along the Middle America Trench reveals significant differences between Cocos-North America and CocosCaribbean convergence: Geophysical Journal International, 166 (1), 179-185, https://doi. org/10.1111/j.1365-246X.2006.02876.x

Guzmán-Speziale, M., Zúñiga, F.R., 2016, Differences and similarities in the CocosNorth America and Cocos-Caribbean convergence, as revealed by seismic moment tensors: Journal of South American Earth Sciences, 71, 296-308. http://dx.doi. org/10.1016/j.jsames.2015.10.002

Hasenaka, T., 1994, Size, distribution, and magma output rate for shield volcanoes of the Michoacán-Guanajuato volcanic field, Central Mexico: Journal of Volcanology and GeothermalResearch,63(1-2),13-31.https:// doi.org/10.1016/0377-0273(94)90016-7

Heidbach, O., Rajabi, M., Reiter, K., Ziegler, M., WSM Team, 2016a, World Stress Map Database Release 2016. V. 1.1. GFZ Data Services. https://doi.org/10.5880/ WSM.2016.001

Heidbach, O., Barth, A., Müller, B., Reinecker, J., Stephansson, O., Tingay, M., Zang, A., 2016b, WSM quality ranking scheme, database description and analysis guidelines for stress indicator: WSM Technical Report, 16-01, Potsdam: GFZ German Research Centre for Geosciences. https://doi. org/10.2312/wsm.2016.001

Herraiz, M., De Vicente, G., Lindo, R., GinerRobles, J.L., Simón, J.L., González- Casado, J.M., Vadillo, O., Rodríguez-Pascua, M.A., Cicuéndez, J., Casas, A., Cabañas, L.,
Rincón Calero, P., Cortes, L., Ramírez, M., Lucini, M., 2000, The recent (upper Miocene to Quaternary) and present tectonic stress distributions in the Iberian Peninsula: Tectonics, 19(4), 762-786. https://doi. org/10.1029/2000TC900006

Husker, A., Davis, P. M., 2009, Tomography and thermal state of the Cocos plate subduction beneath Mexico City: Journal of Geophysical. Research, 114, B04306, https://doi.org/10.1029/2008JB006039

Kagan, Y.Y., 2003, Accuracy of modern global earthquake catalogs: Physics of the Earth and Planetary Interiors, 135, 173-209. https:// doi.org/10.1016/S0031-9201(02)00214-5

Kaverina, A. N., Lander, A.V., Prozorov, A.G., 1996, Global creepex distribution and its relation to earthquake-source geometry and tectonic origin: Geophysical Journal International, 125, (1), 249-265. https://doi. org/10.1111/j.1365-246X.1996.tb06549.x

Kim, Y., Miller, M. S., Pearce, F., Clayton, R. W., 2012, Seismic imaging of the Cocos plate subduction zone system in central Mexico: Geochemistry, Geophysics, Geosystems, 13, Q07001. https://doi. org/10.1029/2012GC004033

Kostoglodov, V., Bandy, W., Domínguez, J., Mena, M., 1996, Gravity and Seismicity over the Guerrero Seismic Gap, Mexico: Geophysical Research Letters, 23, 3385-3388. https:// doi.org/10.1029/96gl03159

Kostoglodov, V., Singh, S. K., Santiago, J. A., Franco, S. I., Larson, K. M., Lowry, A. R., Bilham, R., 2003, A large silent earthquake in the Guerrero seismic gap, Mexico: Geophysical Research Letters, 30, 1807. https://doi.org/10.1029/2003GL017219

Langridge, R. M., Weldon, R. J., Moya, J. C., Suárez, G., 2000, Paleoseismology of the 1912 Acambay earthquake and the Acambay-Tixmadejé fault, Trans-Mexican volcanic belt: Journal of Geophysical Research, 105, 3019-3037. https://doi. org/10.1029/1999JB900239 
León-Soto, G., Ni, J. F., Grand, S. P., Sandvol, E., Valenzuela, R. W., GuzmánSpeziale, M., Gómez-González, J. M., Domínguez-Reyes, T., 2009, Mantle flow in the Rivera-Cocos subduction zone: Geophysical Journal International, 179(2), 1004-1012. https://doi. org/10.1111/j.1365-246X.2009.04352.x

Lee, J.C., Angelier, J., 1994, Paleostress trajectory maps based on the results of local determinations: the "lissage" program, 1994, Computers \& Geosciences, 20(2), 161-191. https://doi. org/10.1016/0098-3004(94)90004-3

Letort, J., Retailleau, L., Boué, P., Radiguet, M., Gardonio, B., Cotton, F., Campillo, M., 2018, Lateral variations of the Guerrero-Oaxaca subduction zone (Mexico) derived from weak seismicity (Mb3.5+) detected on a single array at teleseismic distance: Geophysical Journal International, 213, 1002-1012. https://doi. org/10.1093/gji/ggy035

Lonsdale, P., 1995, Segmentation and Disruption of the East Pacific Rise in the Mouth of the Gulf of California: Marine Geophysical Researches 17, 323-359. https://doi. org/10.1007/BF01227039

Manea V.C., Manea, M., 201 1, Flat-Slab Thermal Structure and Evolution Beneath Central Mexico: Pure and Applied Geophysics, 168, 1475-1487, https://doi.org/10.1007/ s00024-010-0207-9

Manea, V.G., Manea, M., Ferrari, L., OrozcoEsquivel, T., Valenzuela, R.W., Husker, A., Kostoglodov, V., 2017, A review of the geodynamic evolution of flat slab subduction in Mexico, Peru, and Chile: Tectonophysics, 695, 27-52. https://doi.org/10.1016/j. tecto.2016.11.037

Marret, R., Peacock, C.M.P., 1999, Strain and stress: Journal of Structural Geology, 21, 1057-1063. https://doi.org/10.1016/ S0191-8141(99)00020-6

Olaiz, A.J., Muñoz-Martín, A., De Vicente, G., Vegas, R., Cloetingh, S., 2009, European continuous active tectonic strain--stress map:
Tectonophysics, 474, 33-40. https://doi. org/10.1016/j.tecto.2008.06.023

Pardo, M., Suárez, G., 1993, Steep subduction geometry of the Rivera plate beneath the Jalisco block in western Mexico: Geophysical Research Letters, 20 (21), 2391-2394. http:// doi.org/10.1029/93GL02794

Pardo, M., Suárez, G., 1995, Shape of the subducted Rivera and Cocos plates in southern Mexico: Seismic and tectonic implications: Journal of Geophysical Research, 100(B7), 1235712373. https://doi.org/10.1029/95JB00919

Peláez-Gaviria, J.R., Mortera Gutiérrez, C.A., Bandy, W.L., 2013, Morphology and magnetic survey of the Rivera-Cocos plate boundary of Colima, Mexico: Geofísica Internacional, 52(1), 73 - 85. https://doi. org/10.1016/S0016-7169(13)71463-6

Pérez-López R., Giner-Robles, J.L., MartínezDíaz, J.J., Rodríguez-Pascua, M.A., Bejar, M., Paredes C., González-Casado. J.M., 2007, Active strain field on Deception Island (West-Antarctica): a new approach to the collapsed volcanic caldera from the fractal anisotropy of fracture sets. En Cooper, A.K. y Raymond, C.R. (eds.), ANTARCTICA: A Keystone in a Changing World. USGS OpenFile Report 2007-1047, Short Research Paper 086, Proceedings of the 10th ISAES, California - USA.

Pérez-López, R., Mediato, J.F., RodríguezPascua, M.A., Giner-Robles, J.L., Ramos, A., Martín-Velázquez, S., Martínez-Orío, R., Fernández-Canteli, P., 2020, An active tectonic field for $\mathrm{CO}^{2}$ storage management, the Hontomín onshore case study (Spain): Solid Earth, 11(2), 719-739. https://doi. org/10.5194/se-1 1-719-2020

Petricca, P., Carminati, E., 2016, Present-day stress field in subduction zones: Insights from 3D viscoelastic models and data: Tectonophysics, 667, 48-62. https://doi.org/10.1016/j. tecto.2015.11.010

Radiguet, M., Perfettini, H., Cotte, N., Gualandi, A., Valette, B., Kostoglodov, V., Lhomme, T., Walpersdorf, A., Cabral-Cano, E., Campillo, 
M., 2016, Triggering of the 2014 Mw7.3 Papanoa earthquake by a slow slip event in Guerrero, Mexico: Nature Geoscience, 9, 829-833. https://doi.org/10.1038/ ngeo2817

Reches, Z., 1983, Faulting of rocks in threedimensional strain fields II. Theoretical analysis:Tectonophysics, 95,133-156.https:// doi.org/10.1016/0040-1951(83)90264-0

Rodríguez-Pérez, Q., Márquez-Ramírez, V. H., Zúñiga, F. R., 2020, Seismicity characterization of oceanic earthquakes in the Mexican territory: Solid Earth, 11, 791-806. https://doi.org/10.5194/se-11-791-2020

Rodríguez-Pascua, M.A., Pérez-López, R., Garduño-Monroy, V.H., Perucha, M.A., Israde-Alcántara I., 2017, Estimation of the epicentral area of the 1912 Acambay earthquake (M 6.9, Mexico) determined from the earthquake archaeological effects (EAE) and theESI07 macroseismicscale:Quaternary International, 451, 74-86. https://doi. org/10.1016/j.quaint.2017.06.045

Singh, S.K., Pardo, M., 1993, Geometry of the Benioff zone and state of stress in the overriding plate in central Mexico: Geophysical Research Letters, 20(14), 14831486. https://doi.org/10.1029/93gl01310

Suárez, G., Monfret, T., Wittlinger, G., David, G., 1990, Geometry of subduction and depth of the seismogenic zone in the Guerrero Gap, Mexico: Nature, 345, 6273, 336-338. https://doi.org/10.1038/345336a0

Suárez, G., Jaramillo, S.H., Bandy, W.L., 2003, Relative Motion Between the Rivera and North American Plates Determined from the Slip Directions of Earthquakes: Pure and Applied Geophysics, 170, 2163-2172. https://doi.org/10.1007/ s00024-013-0667-9

Suter, M., 1991, State of stress and active deformation in Mexico and western Central America. En Slemmons, D. B., Engdahl, E. R., Zoback, M. D., Blackwell, D. D. (eds.), Neotectonics of North America: Boulder, Colorado, Geological Society of America, Decade Map. https://doi.org/10.1130/ dnag-csms-neo.401

Valentine A.P., Trampert J., 2012, Assessing the uncertainties on seismic source parameters: Towards realistic error estimates for centroidmoment-tensor determinations: Physics of the Earth and Planetary Interiors, 210211, 36-49. https://doi.org/10.1016/j. pepi.2012.08.003

Valenzuela, R. W., León-Soto, G., 2017, Shear Wave Splitting and Mantle Flow in Mexico: What Have we Learned?: Geofísica Internacional, 56-2, 187-217.

Xu,P.,2004,Determination of regionalstresstensors from fault-slip data: Geophysical Journal International, 157, 1316-1330. https://doi. org/10.1111/j.1365-246X.2004.02271.x

Yang,T., Grand,P. S., Wilson,D., Guzman-Speziale, M., Gómez-González, J. M., DomínguezReyes, T., Ni, J., 2009, Seismic structure beneath the Rivera subduction zone from finite-frequency seismic tomography: Journal of Geophysical Research, 114, B01302. https://doi.org/10.1029/2008JB005830 